OPEN ACCESS

Edited by:

Meng Zhou,

Wenzhou Medical University, China

Reviewed by:

Xiangqian Zheng,

Tianjin Medical University Cancer Institute and Hospital, China

Zaki A Sherif,

Howard University, United States

*Correspondence:

Yue Yu

yuyue@tmu.edu.cn

Ran Su

ran.su@tju.edu.cn

${ }^{\dagger}$ These authors have contributed equally to this work

Specialty section:

This article was submitted to Cancer Genetics,

a section of the journal

Frontiers in Oncology

Received: 30 April 2021

Accepted: 31 July 2021

Published: 26 August 2021

Citation:

Tian Y, LiuX, Hu J, Zhang H, Wang B, Li Y, Fu L, Su R and Yu Y (2021) Integrated Bioinformatic Analysis of the Expression and Prognosis of Caveolae-Related Genes in Human Breast Cancer.

Front. Oncol. 11:703501. doi: 10.3389/fonc.2021.703501

\section{Integrated Bioinformatic Analysis of the Expression and Prognosis of Caveolae-Related Genes in Human Breast Cancer}

\author{
Yao Tian ${ }^{1,2,3,4 \dagger}$, Xiaofeng $\mathrm{Liu}^{2,3+}$, Jing Hu ${ }^{1,2,3}$, Huan Zhang ${ }^{2,3}$, Baichuan Wang ${ }^{5,6}$, \\ Yingxi $\mathrm{Li}^{7}, \mathrm{Li} \mathrm{Fu}^{2,3,8}$, Ran Su${ }^{9 *}$ and $\mathrm{Yue} \mathrm{Yu}^{1,2,3^{*}}$ \\ 1 The First Department of Breast Cancer, Tianjin Medical University Cancer Institute and Hospital, Tianjin, China, \\ ${ }^{2}$ Key Laboratory of Breast Cancer Prevention and Therapy, Ministry of Education, Tianjin Medical University Cancer Institute \\ and Hospital, National Clinical Research Center for Cancer, Tianjin, China, ${ }^{3}$ Key Laboratory of Cancer Prevention and \\ Therapy, Tianjin's Clinical Research Center for Cancer, Tianjin Medical University Cancer Institute and Hospital, Tianjin, China, \\ ${ }^{4}$ Department of General Surgery, Tianjin Medical University General Hospital, Tianiin, China, ${ }^{5}$ Anhui Medical University \\ Clinical College of Chest, Hefei, China, ${ }^{6}$ Department of Thoracic Surgery, Anhui Chest Hospital, Hefei, China, \\ 7 Department of Pathogen Biology, School of Basic Medical Sciences, Tianjin Medical University, Tianjin, China, \\ ${ }^{8}$ Department of Breast Cancer Pathology and Research Laboratory, Tianjin Medical University Cancer Institute and Hospital, \\ Tianjin, China, ${ }^{9}$ School of Computer Software, College of Intelligence and Computing, Tianjin University, Tianjin, China
}

Caveolae-related genes, including CAVs that encodes caveolins and CAVINs that encodes caveolae-associated proteins cavins, have been identified for playing significant roles in a variety of biological processes including cholesterol transport and signal transduction, but evidences related to tumorigenesis and cancer progression are not abundant to correlate with clinical characteristics and prognosis of patients with cancer. In this study, we investigated the expression of these genes at transcriptional and translational levels in patients with breast cancer using Oncomine, Gene Expression Profiling Interactive Analysis (GEPIA), cBioPortal databases, and immunohistochemistry of the patients in our hospital. Prognosis of patients with breast cancer based on the expressions of CAVs and CAVINs was summarized using Kaplan-Meier Plotter with their correlation to different subtyping. The relevant molecular pathways of these genes were further analyzed using Kyoto Encyclopedia of Genes and Genomes (KEGG) pathway database and Gene Set Enrichment Analysis (GSEA). Results elucidated that expression levels of CAV1, CAV2, CAVIN1, CAVIN2, and CAVIN3 were significantly lower in breast cancer tissues than in normal samples, while the expression level of CAVIN2 was correlated with advanced tumor stage. Furthermore, investigations on survival of patients with breast cancer indicated outstanding associations between prognosis and CAVIN2 levels, especially for the patients with estrogen receptor positive (ER+) breast cancer. In conclusion, our investigation indicated CAVIN2 is a potential therapeutic target for patients with ER+ breast cancer, which may relate to functions of cancer cell surface receptors and adhesion molecules.

Keywords: CAVs, CAVINs, breast cancer, biomarkers, bioinformatics, prognosis 


\section{INTRODUCTION}

Caveolae are cave-shaped invaginated structures of cell plasma membrane at the range of 50-100 $\mathrm{nm}$ (1). They exist in many cell types and are enriched in adipocytes and endothelial cells $(2,3)$. Within caveolae, caveolins and cavins (caveolae-associated proteins) consist of the key components that involve in a variety of biological processes including signal transduction, endocytosis, cell cycle regulation, and apoptosis (4). It has been reported that the functions of caveolins and cavins are relevant to the progression of malignant tumor, such as prostate cancer $(5,6)$, breast cancer (7-10), lung cancer (11), liver cancer (12), kidney cancer (13), colon cancer (14), and pancreatic cancer $(15,16)$.

Breast cancer is the most common cancer in women worldwide, contributing $11.7 \%$ of the total number of new cancer cases diagnosed in 2020 (17). On the basis of traditional therapeutic methods such as surgery, chemotherapy and radiotherapy, the introduction of molecular subtyping has brought more significant improvement to the precise treatment for breast cancer. It is mainly divided into four subtypes based on the expression of estrogen receptor (ER), progesterone receptor (PR), and human epidermal growth factor receptor 2 (HER2): Luminal A (ER+ and/or PR+ and HER2-), Luminal B (ER+ and/ or PR+ and HER2+), HER2 enriched (ER-, PR-, and HER2+), and basal-like subtype (triple-negative of the above receptors) (18). Patient with Luminal A or B breast cancer can benefit from endocrine therapy by inhibiting ER. Development of anticancer drugs targeting HER2 has also brought better outcomes to those HER2 positives (19). Although the application of novel and advanced treatment has significantly improved the survival of patients with breast cancer, there are still some individual developed distant metastasis at early stage. Meanwhile, the therapeutic effect of triple negative breast cancer has not been significantly improved due to the lack of specific molecular targets (20). Therefore, it is important to identify molecular targets related to the occurrence, progression, metastasis, and prognosis of breast cancer through the existing research data.

Currently, there are seven family members found as caveolins and cavins. They are caveolin-1 (CAV1), caveolin-2 (CAV2), caveolin-3 (CAV3) (21), cavin-1 (CAVIN1, known as polymerase- 1 and transcript release factor, PTRF), cavin-2 (CAVIN2, known as serum deprivation protein response, SDPR), cavin-3 (CAVIN3, known as sdr-related gene product that binds to c-kinase, SRBC), and cavin-4 (CAVIN4, known as muscle-restricted coiled-coil protein, MURC). Caveolins usually play a paradoxical role in the development of diseases, while they are implicated in both tumor suppression and oncogenesis (22, 23). As the principal structural component of caveolae membrane, the ablation of caveolin-1 can induce caveolae loss (24). Caveolin-1 was also reported to associate with breast cancer stem cell enrichment (25). Kang et al. have showed caveolin-1 expression level was a potential indicator for predicting efficacy of docetaxel in the treatment of triple-negative breast cancer (26). Caveolin-2 can directly interact with caveolin-1 (27). Shatseva et al. have reported miR-199a-3p can promote breast cancer cell proliferation through inhibiting caveolin-2 (28), which indicated caveolin-2 as a tumor suppressor gene. It was also necessary for estrogen-dependent breast cancer cell proliferation. Caveolin-3 is predominantly expressed in muscle cells (29). Few studies reported the function caveolin-3 in cancer. Nevertheless, the functions of caveolins in cancer are still under intensive investigations.

Cavins are caveolae-associated proteins and are indispensable for caveolae biogenesis. Similar to caveolins, cavins also have prominently differential tissue distributions. As a resident protein in caveolae, cavin-1 is widely expressed in a variety types of tissues (30). Yi et al. has reported cavin-1 was essential for drug resistance in breast cancer cell (31). Loss of cavin- 1 is also accompanied by destruction of caveolae (32). Cavin-2 is about $20 \%$ structurally similar to cavin- 1 , but the alteration in expression of cavin-2 does not influence the number of caveolae (33). It has been reported to play significant roles in inhibiting cancer cell migration and metastatic potentials when overexpressed (34). A previous study of our group has discovered that cavin-2 depletion can induce epithelialmesenchymal transition in breast cancer cells by activating TGF- $\beta$ signaling pathway (10). Cavin-3 is reported to be relevant to CAV1 during caveolae budding (35). Several studies have emphasized the epigenetic modification of cavin-3 can contribute to the pathogenesis of cancer $(36,37)$, indicating it as a tumor suppressor candidate. Cavin- 4 is only abundant in muscle cells and is able to interact with cavin-2. Faggi et al. has reported cavin-4 is important in the differentiation process of rhabdomyosarcoma in combination with caveolin-3 (38).

To comprehensively investigate how the dysregulation of CAVs and CAVINs levels associate with clinical characteristics of patients with breast cancer, in this study we conducted integrated bioinformatic analysis using online database and analytic tools to explore whether CAVs and CAVINs are relevant to the prognosis of patients with breast cancer and other neighboring signaling pathways involved. Protein level expression of CAVs and CAVINs were validated using realworld immunohistochemistry samples. These results will provide solid evidences for the prediction of prognosis and precise therapy towards breast carcinoma by targeting caveolae-related genes.

\section{MATERIALS AND METHODS}

\section{Ethical Statement}

Benchwork study using tissue samples from patients with breast cancer was approved by the Institutional Review Board of Tianjin Medical University Cancer Institute and Hospital. Relevant investigations were conducted under the principles stated in the Declaration of Helsinki. Studies using datasets were accomplished and retrieved from publications, therefore relevant ethical documents were considered as obtained or approved.

\section{Oncomine Analysis}

We used Oncomine gene expression array datasets (https://www. oncomine.org/resource/login.html) to analyze the transcriptional levels of caveolae-related proteins in different types of cancer. The 
mRNA levels of CAVs and CAVINs in cancer samples from patients were compared with those in normal samples using a Student's $t$ test to generate a $p$ value. Recommended cutoffs of $p$ value and fold change were defined as 0.01 and 2 respectively.

\section{GEPIA Dataset}

We utilized GEPIA (http://gepia.cancer-pku.cn) to further investigate the correlation of gene expression based on RNA sequencing and tissue type or clinical stages of the patients with breast cancer, which is a novel web tool for analyzing RNA-seq data in 9,736 tumors and 8,587 normal samples from the The Cancer Genome Atlas (TCGA) and the Genotype-Tissue Expression (GTEx) projects (39). The individualized studies were conducted under standard processing requirements.

\section{Immunohistochemistry}

Formalin-fixed, paraffin-embedded (FFPE) sections of breast cancer tissues and paired normal samples were subjected to immunostaining with a rabbit monoclonal antibody of CAVs or CAVINs (all from Abcam, UK. Caveolin-1[CAV1], ab32577; Caveolin-2 [CAV2], ab79397; Caveolin-3 [CAV3], ab173575; PTRF [CAVIN1], ab48824; SDPR [CAVIN2], ab76867; SRBC [CAVIN3], ab179923; MURC [CAVIN4], ab121647). In brief, $5-\mu \mathrm{m}$ thick tissue sections were deparaffinized, rehydrated and subjected to antigen retrieval by boiling in sodium citrate buffer (10 mM, pH 6.0). The sections were incubated at $4^{\circ} \mathrm{C}$ overnight with above antibodies at 1:100 dilution, and then exposed with HRP-conjugated secondary antibody at 1:500 dilution (Abcam, UK) followed by covering 3,3'-diaminobenzidine (DAB) (Sigma, USA). After mounted, the slides were visualized under a light microscope (Carl Zeiss, Germany) and images were captured by microscopy camera (Carl Zeiss, Germany).

To quantitatively evaluate the expression levels of each protein in the samples from patients with breast cancer, we calculated percentage of cells stained and the staining intensity, and scored the IHC images using the following criteria: (a) percentage of stained cells: $0(0 \%), 1$ (1\%-25\%), 2 (26\%$50 \%), 3$ (51\%-75\%), and 4 (> 75\%); and (b) staining intensity: 0 (negative staining), 1 (weak staining), 2 (moderate staining), and 3 (strong staining). The multiplier of the scores (0-12) was summarized as scatter plot.

\section{The Cancer Genome Atlas, cBioPortal, GeneMANIA, and STRING}

The Cancer Genome Atlas (TCGA) database has sequencing, clinical information, and pathological results of patients with different types of cancer (40). We used cBioPortal (https://www.cbioportal.org/ results/oncoprint?session_id=5e623de7e4b0ff7ef5fd9620) to further analyze the data from 1,108 cases of breast invasive carcinoma with complete pathological reports in TCGA. Genomic profiles of CAVs and CAVINs were investigated, including mutations, copy number alterations (CNAs) from genomic identification of significant targets in cancer (GISTIC), mRNA expression Z scores (RNA-seq v.2 RSEM), and protein expression $\mathrm{Z}$ scores (reverse phase protein array [RPPA]). Co-expression and network were determined according to the online instructions of cBioPortal. The proteinprotein interaction networks of CAVs and CAVINs were constructed using the online database Gene MANIA (https:// genemania.org/) and STRING (https://string-db.org).

\section{The Kaplan-Meier Plotter}

The online database Kaplan-Meier plotter (http://kmplot.com/ analysis/) was used to investigate the prognosis of certain mRNA expression according to gene expression data and survival information of patients with breast cancer (http://kmplot.com/ analysis/index.php? $\mathrm{p}=$ service $\&$ cancer $=$ breasthttp://kmplot. com/analysis/index.php? $\mathrm{p}=$ service\&cancer=\%20breast) $(41)$. To analyze overall survival (OS) and relapse-free survival (RFS) of the patients, the samples were divided into high expression group and low expression group by the median expression level of the gene. The risk ratio (hazard ratio $[\mathrm{HR}]$ ) with $95 \%$ confidence interval (CI) and $\log \operatorname{rank} p$ value for each predictor were determined to generate Kaplan-Meier plots. In this study, only the probe sets with best JetSet scores for CAVs and CAVINs were selected to generate Kaplan-Meier plots. Number of members was displayed below the main plot.

\section{Gene Set Enrichment Analysis}

Gene Set Enrichment Analysis (GSEA, https://www.gsea-msigdb. $\mathrm{org} /$ ) is a computational method that determines whether a priory defined set of genes shows statistically significant, concordant differences between different phenotypes. GSEA was used to explore pathways and gene sets associated with CAVIN2 in breast cancer. Gene expression profiles of 406 breast cancer samples were downloaded from TCGA dataset. According to the order of expression level of CAVIN2 and the prognosis of cases, the optimal threshold in the ROC curve was divided into HIGH expression group and LOW expression group. GSEA v4.0.3 was used to determine whether the members of the gene set from the MSigDB database are randomly distributed at the top or bottom of the ranking. If most members of a gene set were positively related to the HIGH group, the set was termed associated with LOW group.

\section{RESULTS}

\section{Comparison of Transcriptional Levels of CAVs and CAVINs in Patients With Breast Cancer}

As three CAV proteins and four CAVIN proteins commonly exist in human cells, we firstly compared the transcriptional levels of CAVs and CAVINs in different types of cancer with those in normal tissues using Oncomine database (Figure 1). The expression levels of CAV1 mRNA were significantly downregulated in patients with breast cancer in 31 datasets. In both Sorlie Breast statistics (18) and Sorlie Breast 2 statistics (42), the lower expression of CAV1 was most prominent in fibroadenoma, with a fold change of -14.128 and -12.864 respectively (Table 1). In TCGA breast statistics (40), Curtis Breast Statistics (43), Richardson Breast 2 Statistics (44), and Perou Breast Statistics (45), CAV1 was also less expressed in cancer tissues than in normal tissues, but the most prominent value appeared in ductal breast carcinoma, with a fold change of -11.297, -7.821, -8.398 , and -9.284 respectively (Table 1). CAV2 mRNA was also 


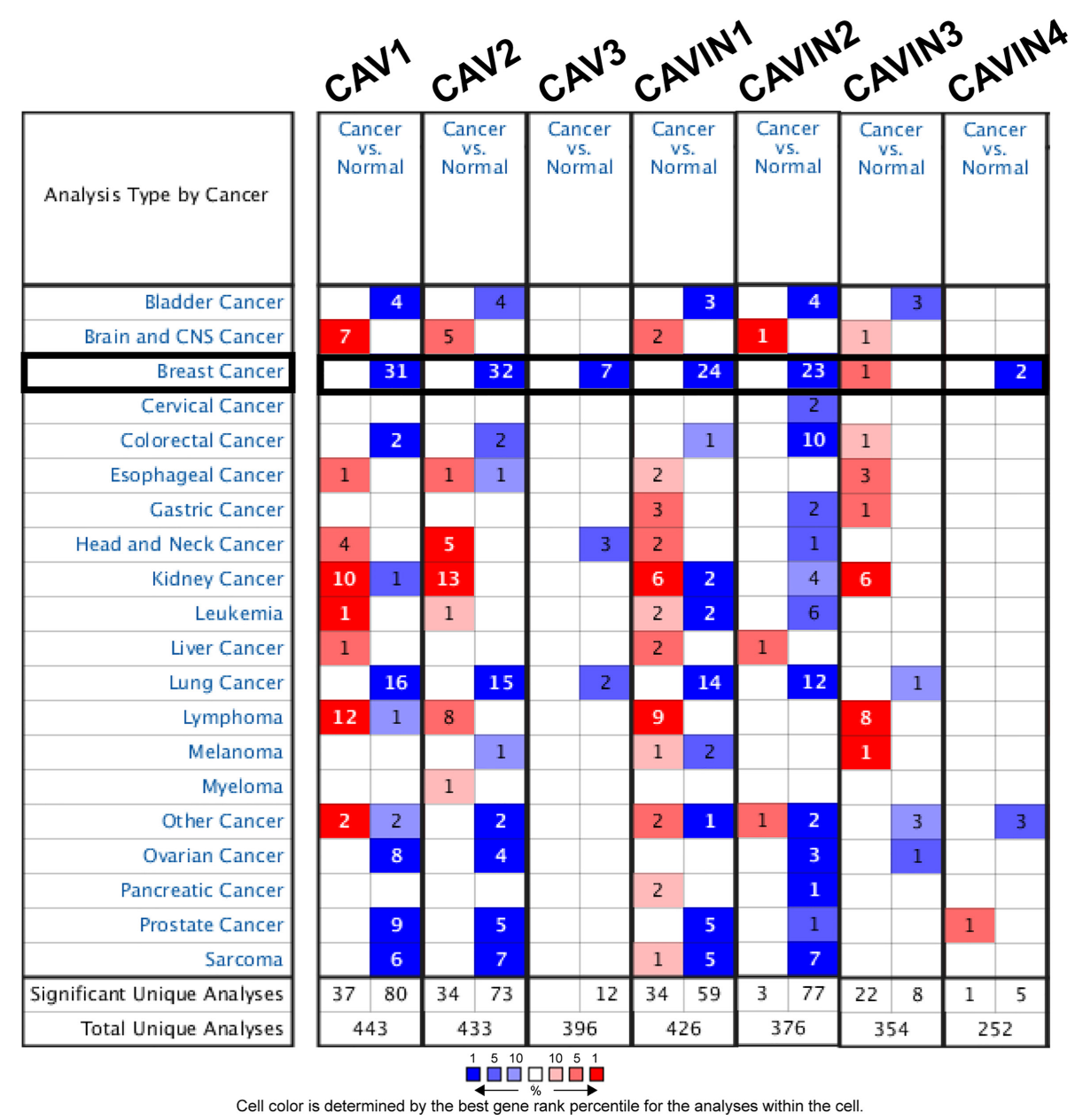

FIGURE 1 | Comparison of transcriptional levels of CAVs and CAVINs in different types of cancers.

found to be lower expressed in cancer tissues in 32 datasets (Figure 1). The most significant fold changes existed in ductal breast carcinoma in most of the datasets except TCGA, which revealed the most significant differences in intraductal cribriform breast adenocarcinoma with a fold change of -11.089 (Table 1). TCGA breast statistics have also showed CAV3 were lower expressed in cancer tissues, with the most prominent fold change of -11.604 in intraductal cribriform breast adenocarcinoma (Table 1).

In terms of CAVIN family, 24 datasets demonstrated lower expression of CAVIN1 in cancer tissues (Figure 1). Perou et al, Zhao et al (46), Richardson et al, Ma et al (47), Sorlie et al, and TCGA all presented the most significant differences in ductal breast cancer (Table 1). The evidences of CAVIN2 lower expression in cancer tissue were provide by 23 datasets (Figure 1), with Ma et al., Radvanyi et al. (48), and Richardson et al. showing the most significant differences in ductal breast cancer. In TCGA, the most prominent downregulation of CAVIN2 existed in intraductal cribriform breast adenocarcinoma with a fold change of -60.805 , which was a similar to the situation of CAV2 and CAV3 (Table 1). CAVIN3 was found higher expressed in invasive breast carcinoma stroma only by Finak et al (49), with a fold change of 3.273. CAVIN4 was found lower expressed by Glück et al (50) and Radvanyi et al, with a fold change of -3.231 in invasive breast cancer and -2.592 in invasive ductal breast cancer respectively.

\section{Relationship Between the Transcriptional and Translational Levels of Caveolae- Related Proteins and Clinicopathological Characteristics of Patients With Breast Cancer}

We then compared the mRNA levels of CAVs and CAVINs in breast cancer tissues and normal ones using the GEPIA (Gene Expression Profiling Interactive Analysis) dataset (39). The results elucidated that the transcriptional levels of CAV1, CAV2, CAVIN1, CAVIN2, and CAVIN3 were significantly lower in breast cancer tissues than in normal samples (Figures 2A, B). However, it should be noticed that the expression levels of CAV3 and CAVIN4 in both cancer tissues 
TABLE 1 | Significant expression changes of caveolae-related proteins in transcription levels between different types of breast cancer and normal breast tissues (Oncomine Database).

\begin{tabular}{|c|c|c|c|c|c|c|}
\hline & $\begin{array}{c}\text { Type of Breast Cancer versus } \\
\text { Normal Breast Tissue }\end{array}$ & $\begin{array}{l}\text { Fold } \\
\text { Change }\end{array}$ & p Value & t Test & Source and/or Reference & \\
\hline \multirow[t]{31}{*}{ CAV1 } & Ductal Breast Carcinoma & -5.462 & $9.14 \mathrm{E}-27$ & -17.953 & Sorlie Breast 2 Statistics & PMID:12829800 \\
\hline & Fibroadenoma & -12.864 & 4.72E-04 & -6.958 & Sorlie Breast 2 Statistics & \\
\hline & Lobular Breast Carcinoma & -6.906 & 3.00E-03 & -3.655 & Sorlie Breast 2 Statistics & \\
\hline & Fibroadenoma & -14.128 & 5.10E-04 & -6.939 & Sorlie Breast Statistics & PMID:11553815 \\
\hline & Ductal Breast Carcinoma & -5.473 & 2.01E-06 & -10.028 & Sorlie Breast Statistics & \\
\hline & Lobular Breast Carcinoma & -12.424 & 7.00E-03 & -3.94 & Sorlie Breast Statistics & \\
\hline & Invasive Breast Carcinoma & -7.607 & $1.92 E-35$ & -17.06 & TCGA Breast Statistics & TCGA \\
\hline & Invasive Lobular Breast Carcinoma & -6.366 & 8.58E-20 & -12.522 & TCGA Breast Statistics & \\
\hline & Mixed Lobular and Ductal Breast Carcinoma & -4.73 & 8.31E-12 & -10.033 & TCGA Breast Statistics & \\
\hline & Invasive Ductal Breast Carcinoma & -11.297 & $1.00 E-40$ & -26.387 & TCGA Breast Statistics & \\
\hline & Mucinous Breast Carcinoma & -5.711 & 7.32E-06 & -9.074 & TCGA Breast Statistics & \\
\hline & Invasive Ductal and Lobular Carcinoma & -8.837 & 9.05E-05 & -12.806 & TCGA Breast Statistics & \\
\hline & Male Breast Carcinoma & -8.963 & 1.14E-04 & -12.654 & TCGA Breast Statistics & \\
\hline & Intraductal Cribriform Breast Adenocarcinoma & -5.777 & 1.00E-03 & -7.544 & TCGA Breast Statistics & \\
\hline & Mucinous Breast Carcinoma & -4.257 & 3.94E-37 & -17.398 & Curtis Breast Statistics & PMID:22522925 \\
\hline & Tubular Breast Carcinoma & -3.784 & $6.91 E-45$ & -18.091 & Curtis Breast Statistics & \\
\hline & Invasive Lobular Breast Carcinoma & -4.9 & 2.23E-60 & -21.145 & Curtis Breast Statistics & \\
\hline & $\begin{array}{l}\text { Invasive Ductal and Invasive Lobular Breast } \\
\text { Carcinoma }\end{array}$ & -5.4 & $1.68 E-48$ & -20.446 & Curtis Breast Statistics & \\
\hline & Invasive Ductal Breast Carcinoma & -7.821 & 8.68E-91 & -41.04 & Curtis Breast Statistics & \\
\hline & Medullary Breast Carcinoma & -4.592 & $6.01 E-28$ & -16.472 & Curtis Breast Statistics & \\
\hline & Breast Carcinoma & -4.565 & 8.39E-13 & -13.211 & Curtis Breast Statistics & \\
\hline & Invasive Breast Carcinoma & -4.065 & 1.17E-12 & -10.928 & Curtis Breast Statistics & \\
\hline & Benign Breast Neoplasm & -2.73 & 2.84E-04 & -10.894 & Curtis Breast Statistics & \\
\hline & Ductal Breast Carcinoma In situ & -3.55 & 5.57E-06 & -7.332 & Curtis Breast Statistics & \\
\hline & Breast Phyllodes Tumor & -4.268 & 6.00E-03 & -4.352 & Curtis Breast Statistics & \\
\hline & Invasive Breast Carcinoma Stroma & -31.965 & $2.62 E-31$ & -23.515 & Finak Breast Statistics & PMID:18438415 \\
\hline & Invasive Ductal Breast Carcinoma Stroma & -2.077 & 5.01E-05 & -5.808 & Ma Breast 4 Statistics & PMID:19187537 \\
\hline & Ductal Breast Carcinoma In situ Epithelia & -4.516 & 1.28E-05 & -5.477 & Ma Breast 4 Statistics & \\
\hline & Invasive Ductal Breast Carcinoma Epithelia & -3.683 & 8.00E-03 & -2.759 & Ma Breast 4 Statistics & \\
\hline & Ductal Breast Carcinoma & -8.398 & $2.42 \mathrm{E}-09$ & -10.429 & $\begin{array}{l}\text { Richardson Breast } 2 \\
\text { Statistics }\end{array}$ & PMID:16473279 \\
\hline & Ductal Breast Carcinoma & -9.284 & 5.00E-03 & -7.084 & Perou Breast Statistics & PMID:10963602 \\
\hline \multirow[t]{27}{*}{ CAV2 } & Fibroadenoma & -5.489 & 1.00E-03 & -6.986 & Sorlie Breast Statistics & PMID:11553815 \\
\hline & Lobular Breast Carcinoma & -5.038 & 5.00E-03 & -4.514 & Sorlie Breast Statistics & \\
\hline & Ductal Breast Carcinoma & -5.714 & 1.00E-03 & -7.357 & Sorlie Breast Statistics & \\
\hline & Invasive Lobular Breast Carcinoma & -4.797 & $1.12 \mathrm{E}-19$ & -12.538 & TCGA Breast Statistics & TCGA \\
\hline & Mixed Lobular and Ductal Breast Carcinoma & -6.152 & 8.77E-12 & -12.276 & TCGA Breast Statistics & \\
\hline & Invasive Breast Carcinoma & -4.587 & 1.19E-30 & -14.97 & TCGA Breast Statistics & \\
\hline & Invasive Ductal Breast Carcinoma & -7.006 & $3.54 \mathrm{E}-41$ & -24.744 & TCGA Breast Statistics & \\
\hline & Mucinous Breast Carcinoma & -9.574 & 2.47E-05 & -13.146 & TCGA Breast Statistics & \\
\hline & Male Breast Carcinoma & -6.681 & 2.33E-05 & -11.408 & TCGA Breast Statistics & \\
\hline & Intraductal Cribriform Breast Adenocarcinoma & -11.089 & 3.00E-03 & -7.953 & TCGA Breast Statistics & \\
\hline & Invasive Ductal and Lobular Carcinoma & -7.218 & 2.00E-03 & -7.867 & TCGA Breast Statistics & \\
\hline & Fibroadenoma & -4.998 & 1.00E-03 & -6.617 & Sorlie Breast 2 Statistics & PMID:12829800 \\
\hline & Lobular Breast Carcinoma & -4.287 & 1.00E-03 & -4.723 & Sorlie Breast 2 Statistics & \\
\hline & Ductal Breast Carcinoma & -5.787 & 1.00E-03 & -7.653 & Sorlie Breast 2 Statistics & \\
\hline & Invasive Ductal and Invasive Lobular Breast Carcinoma & -4.241 & $1.92 \mathrm{E}-51$ & -20.149 & Curtis Breast Statistics & PMID:22522925 \\
\hline & Breast Carcinoma & -2.111 & $1.04 \mathrm{E}-17$ & -13.168 & Curtis Breast Statistics & \\
\hline & Mucinous Breast Carcinoma & -2.069 & 8.17E-35 & -15.247 & Curtis Breast Statistics & \\
\hline & Ductal Breast Carcinoma In situ & -2.025 & $5.38 \mathrm{E}-10$ & -10.403 & Curtis Breast Statistics & \\
\hline & Tubular Breast Carcinoma & -3.93 & $5.76 \mathrm{E}-43$ & -18.687 & Curtis Breast Statistics & \\
\hline & Invasive Breast Carcinoma & -2.036 & 2.87E-15 & -11.012 & Curtis Breast Statistics & \\
\hline & Invasive Lobular Breast Carcinoma & -3.761 & $3.90 E-55$ & -19.555 & Curtis Breast Statistics & \\
\hline & Medullary Breast Carcinoma & -2.1 & $2.57 \mathrm{E}-24$ & -13.222 & Curtis Breast Statistics & \\
\hline & Invasive Ductal Breast Carcinoma & -5.178 & $4.42 \mathrm{E}-73$ & -32.597 & Curtis Breast Statistics & \\
\hline & Breast Phyllodes Tumor & -3.494 & 3.67E-04 & -7.126 & Curtis Breast Statistics & \\
\hline & Invasive Mixed Breast Carcinoma & -3.272 & 2.00E-03 & -4.346 & Radvanyi Breast Statistics & PMID:16043716 \\
\hline & Invasive Lobular Breast Carcinoma & -2.624 & $5.00 \mathrm{E}-03$ & -3.207 & Radvanyi Breast Statistics & \\
\hline & Invasive Ductal Breast Carcinoma & -2.346 & 8.00E-03 & -2.888 & Radvanyi Breast Statistics & \\
\hline
\end{tabular}


TABLE 1 | Continued

\begin{tabular}{|c|c|c|c|c|c|c|}
\hline & $\begin{array}{c}\text { Type of Breast Cancer versus } \\
\text { Normal Breast Tissue }\end{array}$ & $\begin{array}{c}\text { Fold } \\
\text { Change }\end{array}$ & p Value & t Test & Source and/or Reference & \\
\hline & Lobular Breast Carcinoma & -3.759 & 3.00E-03 & -4.583 & Perou Breast Statistics & PMID:10963602 \\
\hline & Ductal Breast Carcinoma & -5.945 & 1.00E-03 & -9.231 & Perou Breast Statistics & \\
\hline & Ductal Breast Carcinoma & -5.031 & $6.00 \mathrm{E}-10$ & -9.235 & $\begin{array}{l}\text { Richardson Breast } 2 \\
\text { Statistics }\end{array}$ & PMID:16473279 \\
\hline & Invasive Breast Carcinoma & -10.091 & 1.44E-04 & -13.884 & Glück Breast Statistics & PMID:21373875 \\
\hline & Invasive Ductal Breast Carcinoma Epithelia & -4.693 & 7.00E-03 & -2.961 & Ma Breast 4 Statistics & PMID:19187537 \\
\hline \multirow[t]{7}{*}{ CAV3 } & Invasive Lobular Breast Carcinoma & -4.6 & 2.34E-22 & -12.958 & TCGA Breast Statistics & TCGA \\
\hline & Invasive Breast Carcinoma & -5.762 & $6.70 E-34$ & -16.936 & TCGA Breast Statistics & \\
\hline & Mixed Lobular and Ductal Breast Carcinoma & -5.692 & $1.41 \mathrm{E}-10$ & -13.479 & TCGA Breast Statistics & \\
\hline & Invasive Ductal Breast Carcinoma & -7.809 & 2.49E-36 & -24.176 & TCGA Breast Statistics & \\
\hline & Mucinous Breast Carcinoma & -6.402 & $1.42 \mathrm{E}-04$ & -9.903 & TCGA Breast Statistics & \\
\hline & Invasive Ductal and Lobular Carcinoma & -7.293 & 1.38E-04 & -12.868 & TCGA Breast Statistics & \\
\hline & Intraductal Cribriform Breast Adenocarcinoma & -11.604 & $5.00 \mathrm{E}-03$ & -7.772 & TCGA Breast Statistics & \\
\hline \multirow[t]{24}{*}{ Cavin-1/PTRF } & Invasive Ductal Breast Carcinoma & -2.739 & $\begin{array}{c}\text { 1.03E- } \\
122\end{array}$ & -42.749 & Curtis Breast Statistics & PMID:22522925 \\
\hline & Invasive Lobular Breast Carcinoma & -2.46 & $6.01 E-46$ & -18.531 & Curtis Breast Statistics & \\
\hline & $\begin{array}{l}\text { Invasive Ductal and Invasive Lobular Breast } \\
\text { Carcinoma }\end{array}$ & -2.628 & 1.67E-33 & -17.036 & Curtis Breast Statistics & \\
\hline & Medullary Breast Carcinoma & -3.31 & $4.20 \mathrm{E}-13$ & -11.173 & Curtis Breast Statistics & \\
\hline & Mucinous Breast Carcinoma & -3.539 & 1.09E-18 & -13.639 & Curtis Breast Statistics & \\
\hline & Breast Carcinoma & -3.23 & 7.98E-07 & -8.061 & Curtis Breast Statistics & \\
\hline & Tubular Breast Carcinoma & -2.23 & 3.64E-25 & -14.495 & Curtis Breast Statistics & \\
\hline & Ductal Breast Carcinoma In situ & -2.645 & 3.36E-04 & -5.023 & Curtis Breast Statistics & \\
\hline & Invasive Breast Carcinoma & -2.764 & 2.26E-05 & -5.155 & Curtis Breast Statistics & \\
\hline & Ductal Breast Carcinoma & -4.015 & 1.86E-06 & -11.704 & Perou Breast Statistics & PMID:10963602 \\
\hline & Lobular Breast Carcinoma & -2.784 & 8.00E-03 & -4.216 & Perou Breast Statistics & \\
\hline & Invasive Ductal Breast Carcinoma & -2.362 & 1.59E-09 & -8.105 & Zhao Breast Statistics & PMID:15034139 \\
\hline & Ductal Breast Carcinoma & -5.807 & $1.24 \mathrm{E}-11$ & -12.161 & $\begin{array}{l}\text { Richardson Breast } 2 \\
\text { Statistics }\end{array}$ & PMID:16473279 \\
\hline & Ductal Breast Carcinoma In situ Epithelia & -5.638 & 1.26E-06 & -8.205 & Ma Breast 4 Statistics & PMID:19187537 \\
\hline & Lobular Breast Carcinoma & -2.974 & 9.00E-03 & -3.765 & Sorlie Breast Statistics & PMID:11553815 \\
\hline & Ductal Breast Carcinoma & -4.216 & 7.82E-05 & -10.649 & Sorlie Breast Statistics & \\
\hline & Lobular Breast Carcinoma & -2.334 & 3.00E-03 & -3.551 & Sorlie Breast 2 Statistics & PMID:12829800 \\
\hline & Ductal Breast Carcinoma & -4.014 & 1.07E-04 & -11.117 & Sorlie Breast 2 Statistics & \\
\hline & Intraductal Cribriform Breast Adenocarcinoma & -3.105 & $7.68 \mathrm{E}-12$ & -10.555 & TCGA Breast Statistics & TCGA \\
\hline & Mucinous Breast Carcinoma & -2.357 & 3.59E-07 & -7.073 & TCGA Breast Statistics & \\
\hline & Invasive Breast Carcinoma & -3.21 & $6.66 \mathrm{E}-21$ & -11.201 & TCGA Breast Statistics & \\
\hline & Mixed Lobular and Ductal Breast Carcinoma & -2.766 & 7.52E-06 & -6.982 & TCGA Breast Statistics & \\
\hline & Invasive Lobular Breast Carcinoma & -2.862 & 1.14E-12 & -8.111 & TCGA Breast Statistics & \\
\hline & Invasive Ductal Breast Carcinoma & -4.04 & 1.48E-27 & -16.278 & TCGA Breast Statistics & \\
\hline \multirow[t]{21}{*}{ Cavin-2/SDPR } & Invasive Lobular Breast Carcinoma & -4.857 & 4.64E-81 & -27.075 & Curtis Breast Statistics & PMID:22522925 \\
\hline & Tubular Breast Carcinoma & -5.474 & $6.55 E-64$ & -26.805 & Curtis Breast Statistics & \\
\hline & Medullary Breast Carcinoma & -8.162 & $3.28 E-56$ & -33.594 & Curtis Breast Statistics & \\
\hline & $\begin{array}{l}\text { Invasive Ductal and Invasive Lobular Breast } \\
\text { Carcinoma }\end{array}$ & -5.102 & $3.45 E-63$ & -24.597 & Curtis Breast Statistics & \\
\hline & Invasive Ductal Breast Carcinoma & -7.136 & $6.27 E-90$ & -44.083 & Curtis Breast Statistics & \\
\hline & Mucinous Breast Carcinoma & -5.711 & 1.73E-31 & -19.506 & Curtis Breast Statistics & \\
\hline & Breast Carcinoma & -6.516 & $1.35 E-13$ & -17.841 & Curtis Breast Statistics & \\
\hline & Invasive Breast Carcinoma & -6.158 & $6.91 \mathrm{E}-13$ & -13.096 & Curtis Breast Statistics & \\
\hline & Ductal Breast Carcinoma In situ & -4.993 & 1.93E-07 & -10.85 & Curtis Breast Statistics & \\
\hline & Breast Phyllodes Tumor & -3.766 & 6.00E-03 & -4.255 & Curtis Breast Statistics & \\
\hline & Invasive Ductal Breast Carcinoma Stroma & -11.593 & 5.95E-07 & -7.798 & Ma Breast 4 Statistics & PMID:19187537 \\
\hline & Ductal Breast Carcinoma In situ Epithelia & -2.864 & 4.81E-05 & -5.169 & Ma Breast 4 Statistics & \\
\hline & Ductal Breast Carcinoma In situ Stroma & -3.589 & 5.31E-04 & -3.914 & Ma Breast 4 Statistics & \\
\hline & Invasive Breast Carcinoma & -14.278 & $3.70 \mathrm{E}-33$ & -16.431 & TCGA Breast Statistics & TCGA \\
\hline & Mucinous Breast Carcinoma & -24.174 & 2.32E-14 & -23.228 & TCGA Breast Statistics & \\
\hline & Invasive Ductal Breast Carcinoma & -25.347 & 2.29E-55 & -32.344 & TCGA Breast Statistics & \\
\hline & Invasive Lobular Breast Carcinoma & -10.124 & $1.16 \mathrm{E}-17$ & -12.301 & TCGA Breast Statistics & \\
\hline & Mixed Lobular and Ductal Breast Carcinoma & -15.494 & 1.28E-05 & -8.897 & TCGA Breast Statistics & \\
\hline & Intraductal Cribriform Breast Adenocarcinoma & -60.805 & 1.00E-03 & -12.429 & TCGA Breast Statistics & \\
\hline & Invasive Ductal and Lobular Carcinoma & -12.004 & 3.00E-03 & -9.072 & TCGA Breast Statistics & \\
\hline & Invasive Breast Carcinoma & -18.248 & 8.84E-06 & -17.025 & Glück Breast Statistics & PMID:21373875 \\
\hline
\end{tabular}

(Continued) 
TABLE 1 | Continued

\begin{tabular}{|c|c|c|c|c|c|c|}
\hline & $\begin{array}{c}\text { Type of Breast Cancer versus } \\
\text { Normal Breast Tissue }\end{array}$ & $\begin{array}{c}\text { Fold } \\
\text { Change }\end{array}$ & p Value & t Test & Source and/or Reference & \\
\hline & Invasive Ductal Breast Carcinoma & -6.155 & 2.00E-03 & -3.749 & Radvanyi Breast Statistics & PMID:16043716 \\
\hline & Ductal Breast Carcinoma & -4.895 & 1.43E-04 & -6.997 & $\begin{array}{l}\text { Richardson Breast } 2 \\
\text { Statistics }\end{array}$ & PMID:16473279 \\
\hline $\begin{array}{l}\text { Cavin-3/SRBC/ } \\
\text { PRKCDBP }\end{array}$ & Invasive Breast Carcinoma Stroma & 3.273 & $3.83 E-16$ & 13.421 & Finak Breast Statistics & PMID:18438415 \\
\hline \multirow[t]{2}{*}{ Cavin-4/MURC } & Invasive Breast Carcinoma & -3.231 & $1.28 \mathrm{E}-10$ & -18.542 & Glück Breast Statistics & PMID:21373875 \\
\hline & Invasive Ductal Breast Carcinoma & -2.592 & 7.39E-04 & -4.158 & Radvanyi Breast Statistics & PMID:16043716 \\
\hline
\end{tabular}

and normal tissues were rather low (Figure 2B), indicating a limited role of these two proteins in the tumorigenesis and progression of breast cancer. Furthermore, the expression levels of these caveolae-related genes were further correlated with cancer stage of the patients. We found that CAV3, CAVIN2, and CAVIN4 were significantly differed among stages, while others did not show differences (Figure 2C).

To validate the results from database, we have conducted immunohistochemistry (IHC) to verify the expression status of CAVs and CAVINs in breast cancer tissues and paired normal samples from patients in our hospital. 20 paired samples from patients with breast cancer were involved in IHC experiments.
The statistical results showed that CAV1, CAV2, CAV3, CAVIN1, and CAVIN2 were significantly less expressed in breast cancer tissues than in normal ones (Figure 3). Taken together, differences in expression level of CAVIN2 was the most prominent of all the caveolae-related genes.

We then used cBioPortal (The Cancer Genome Atlas, Provisional; https://www.cbioportal.org/results/oncoprint? session_id=5e623de7e4b0ff7ef5fd9620) to investigate gene alterations, correlations and networks of CAVs and CAVINs in invasive breast cancer. Results showed that CAVs and CAVINs were altered in 289 samples of 1,108 patients with breast invasive carcinoma (26\%) (Figure 4A). Among these caveolae-related

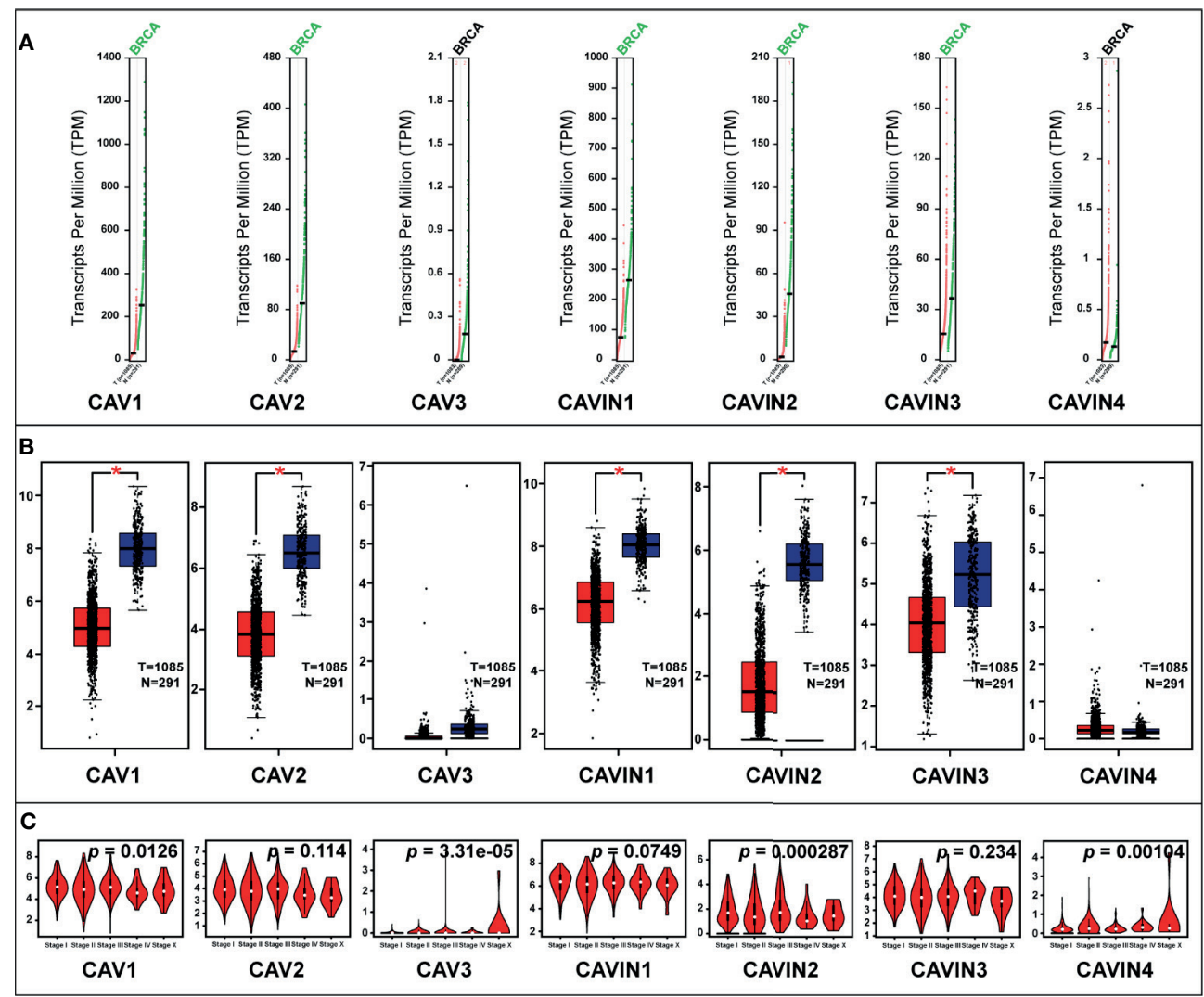

FIGURE 2 | (A) Scatter diagram of expressions of CAVs and CAVINs in breast cancer. (B) Box plot of expression of CAVs and CAVINs in breast cancer ( $\left.{ }^{\star} p<0.05\right)$. (C) Correlation between CAVs and CAVINs levels and clinical stages of patients with breast cancer. 


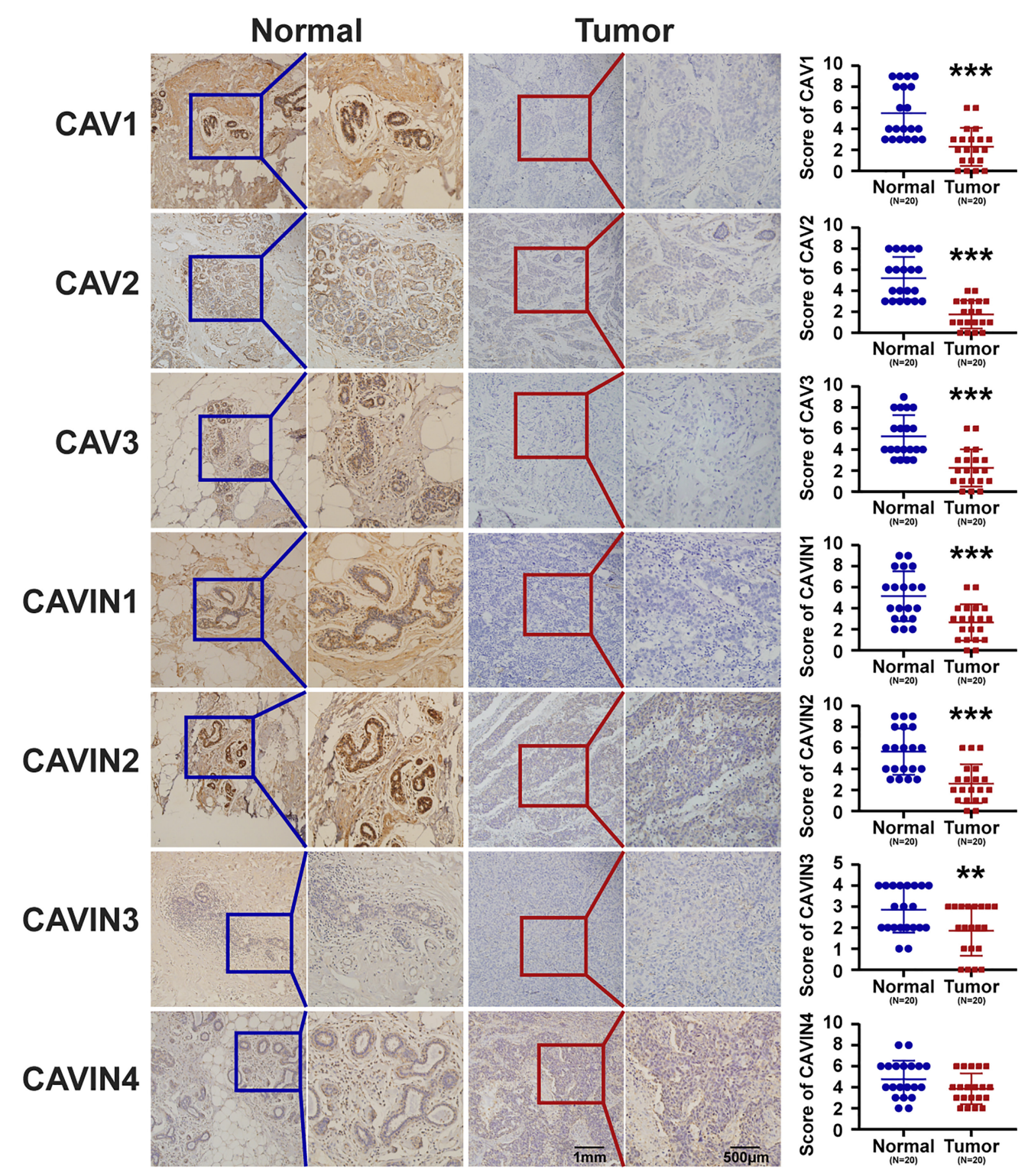

FIGURE 3 | Representative immunohistochemical images of CAVs and CAVINs in cancer tissues with paired normal samples from patients with breast cancer. 20 samples were involved for $\mathrm{HC}$ analysis and the corresponding $\mathrm{HC}$ scores were summarized as scatter plots on the right. $\left({ }^{* \star} p<0.01,{ }^{* \star *} p<0.005\right)$.

genes, CAV1 achieved the highest alteration rate at $9 \%$, of which the majority were mRNA dysregulation and amplification (Figure 4A). CAVIN1 and CAVIN2 achieved the highest rate of mRNA downregulation, consisting of more than half the alterations (Figure 4A). Furthermore, the correlations of CAVs and CAVINs were calculated by analyzing their mRNA expression with the inclusion of Pearson's correction. The results showed that there were significant and positive correlations in the following CAVs and CAVINs: CAV1 with CAV2, CAVIN1, CAVIN2, and CAVIN3; CAV2 with CAV1, CAVIN1, and CAVIN2; CAV3 with CAVIN4; CAVIN1 with CAV1, CAV2, CAVIN2, and CAVIN3; CAVIN2 with CAV1,
CAV2 and CAVIN1; CAVIN3 with CAV1 and CAVIN1; and CAVIN4 with CAV3 (Figure 4B). The molecular interaction network was then constructed for CAVs, CAVINs and other most frequently changed neighbor genes with the application of GeneMANIA database (https://genemania.org/) and STRING database (https://string-db.org/cgi/network.pl?taskId= TPhhdLzBayql). In GeneMANIA analysis, results showed strong associations between CAV1, CAVIN1 (PTRF), CAVIN2 (SDPR), and the most prominent weight of SCP2, HMCN1, MYRIP, and AP3B1, suggesting (Figure 4C). According to STRING database, our results elucidated that the adhesionrelated genes, including SHC1, EGFR, SRC, EGF, FYN, and 


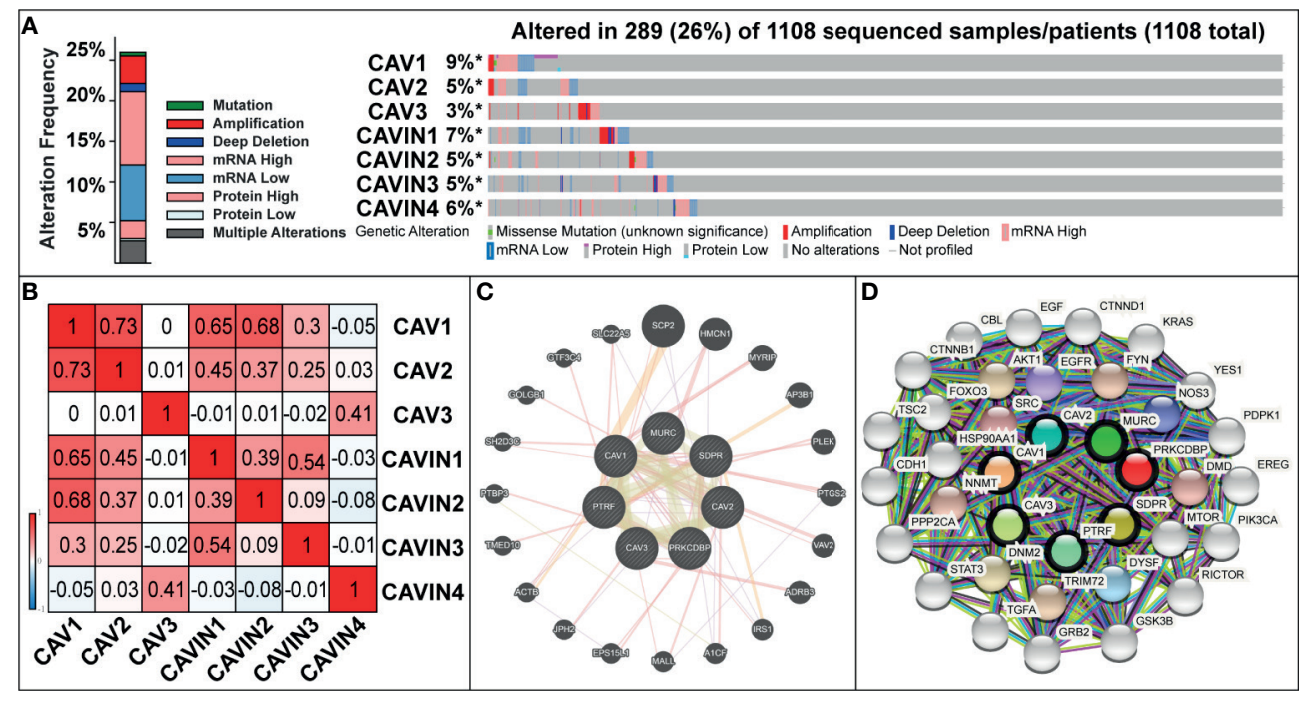

FIGURE 4 | (A) Gene mutation analysis of CAVs and CAVINs in patients with breast cancer. (B) Pearson's correction between CAVs and CAVINs. (C) GeneMANIA analysis of relevant interactive genes of CAVs and CAVINs. (D) Molecular network for CAVs and CAVINs and 37 most frequently altered neighbor genes.

PI3K-Akt signaling pathway-related genes, including FOXO3, NOS3, EGFR, HSP90AA1, EGF, were significantly associated with caveolins and cavins alterations (Figure 4D).

\section{Relationship Between the Expression of CAVs and CAVINs and Prognosis of Patients With Breast Cancer}

Next, we investigated the associations of CAVs and CAVINs levels with survival of the patients. We used Kaplan-Meier Plotter (41) to generate Kaplan Meier survival curve with log rank test under a variety of grouping conditions. Overall survival (OS), relapse-free survival (RFS), distant metastasis-free survival (DMFS), and post progression survival (PPS) were summarized as shown in Tables 2-4 and Figures S1-S3. Results indicated that in all the population of patients with breast cancer in the database, decreased levels of CAV1, CAVIN1 and CAVIN2 were statistically related to poor OS of patients with breast cancer $(p<0.05)$ (Table 2 and Figure S1), and lower expression of CAV1, CAV2, CAV3, CAVIN1, CAVIN2, and CAVIN4 were statistically related to shorter relapse-free survival (RFS) of the patients $(p<0.05)$ (Table 2 and Figure S1). Among these curves, CAVIN1 and CAVIN2 levels achieved the most remarkable capabilities in distinguishing OS, while CAV3 and CAVIN2 achieved the most in distinguishing RFS.

Then we investigated whether CAVs and CAVINs levels significantly affected prognosis of patients with different molecular subtypes of breast cancer. Our results elucidated that lower expression of CAV3, CAVIN1, and CAVIN2 significantly related to OS of patients with Luminal A breast cancer (Table 3 and Figure S2). Protein levels of all CAVs, CAVIN1, and CAVIN2 were associated with RFS of patients with Luminal A breast cancer, suggesting these caveolae-related genes can be potentially independent predictive factors of the prognosis of
Luminal A breast cancer (Table 3 and Figure S2). Remarkably, lower expression levels of CAV1, CAV2, and CAVIN3 were significantly relevant to better RFS of patients with basal-like breast cancer, indicating in triple-negative breast cancer, caveolae-related genes may play different roles in carcinogenesis and tumor progression as them in hormone receptor-positive breast cancer (Table 3 and Figure S2).

Furthermore, the associations between protein levels and survival of patients was the most obvious in the KM curves revealing the expression of CAVIN2 and RFS of HER2 negative, progesterone receptor (PR) positive, and estrogen receptor (ER) positive breast cancer (Table 4 and Figure S3). CAV1 and CAV2 also elucidated independent predicting value in the RFS of ER+ breast cancer patients, but the prognosis of breast cancer patients with other receptor status was not well differentiated by expression levels of CAV1 and CAV2 (Table 4 and Figure S3). In conclusion, CAVIN2 achieved the highest potential of independently predicting prognosis of patients with ER+ breast cancer among all the caveolae-related genes.

Based on the above investigations, we further cross-analyzed the RFS of patients with breast cancer for intra-family study. The comparison within CAV family showed that no matter how much CAVs expressed, the transcriptional level of CAV3 can always distinguish RFS of patients with statistically differences, and the similar situation occurred on CAVIN2 from CAVINs family as shown in Figure S4. Taken together, these results revealed a remarkable prognostic potential of CAVIN2 in breast cancer populations.

\section{Prediction of Functions and Related Signaling Pathway of CAVs and CAVINs in Patients With Breast Cancer}

To predict the function of CAVs and CAVINs and the genes significantly related to the alterations of them, we investigated 
TABLE 2 | Correlation of CAVs/CAVINs transcription levels and survivals of patients with breast cancer.

\begin{tabular}{|c|c|c|c|c|c|c|}
\hline Gene & Dataset/ Affymetrix ID & Survival outcome & No.of cases & HR & $95 \% \mathrm{Cl}$ & $p$-value \\
\hline \multirow[t]{4}{*}{ CAV1 } & 212097_at & OS & 1402 & 0.81 & 0.65-1 & 0.047 \\
\hline & & RFS & 3951 & 0.87 & $0.78-0.97$ & 0.012 \\
\hline & & DMFS & 1803 & 0.91 & $0.76-1.11$ & 0.36 \\
\hline & & PPS & 414 & 0.97 & $0.76-1.24$ & 0.81 \\
\hline \multirow[t]{4}{*}{ CAV2 } & 203323_at & OS & 1402 & 0.89 & $0.72-1.1$ & 0.29 \\
\hline & & RFS & 3951 & 0.88 & $0.79-0.98$ & 0.021 \\
\hline & & DMFS & 1803 & 1.02 & $0.84-1.23$ & 0.84 \\
\hline & & PPS & 414 & 1.08 & $0.85-1.38$ & 0.52 \\
\hline \multirow[t]{4}{*}{ CAV3 } & 208204_s_at & OS & 1402 & 0.82 & $0.66-1.01$ & 0.067 \\
\hline & & RFS & 3951 & 0.75 & $0.67-0.84$ & 2.2E-07 \\
\hline & & DMFS & 1803 & 0.92 & $0.76-1.11$ & 0.37 \\
\hline & & PPS & 414 & 0.87 & $0.68-1.11$ & 0.25 \\
\hline \multirow[t]{4}{*}{ CAVIN1 } & 208789_at & OS & 1402 & 0.75 & $0.6-0.92$ & 0.0073 \\
\hline & & RFS & 3951 & 0.82 & $0.74-0.91$ & 0.00035 \\
\hline & & DMFS & 1803 & 1.03 & $0.85-1.25$ & 0.77 \\
\hline & & PPS & 414 & 0.87 & $0.69-1.11$ & 0.27 \\
\hline \multirow[t]{4}{*}{ CAVIN2 } & 222717_at & OS & 626 & 0.66 & $0.48-0.9$ & 0.0087 \\
\hline & & RFS & 1764 & 0.65 & $0.55-0.76$ & 4.6E-08 \\
\hline & & DMFS & 664 & 0.78 & $0.57-1.08$ & 0.14 \\
\hline & & PPS & 173 & 1.29 & $0.91-1.84$ & 0.15 \\
\hline \multirow[t]{4}{*}{ CAVIN3 } & 213010_at & OS & 1402 & 1 & $0.8-1.23$ & 0.97 \\
\hline & & RFS & 3951 & 1.04 & $0.93-1.16$ & 0.46 \\
\hline & & DMFS & 1803 & 1.11 & $0.92-1.34$ & 0.29 \\
\hline & & PPS & 414 & 1.02 & 0.8-1.3 & 0.88 \\
\hline \multirow[t]{4}{*}{ CAVIN4 } & 241749_at & OS & 626 & 1.11 & $0.81-1.51$ & 0.52 \\
\hline & & RFS & 1764 & 1.25 & $1.07-1.46$ & 0.0045 \\
\hline & & DMFS & 664 & 1.38 & $1-1.91$ & 0.051 \\
\hline & & PPS & 173 & 0.82 & $0.57-1.17$ & 0.27 \\
\hline
\end{tabular}

Bold values mean $p$ value less than 0.05 .

TABLE 3 | Correlation of CAVs/CAVINs transcription levels and survivals of patients with different molecular subtypes of breast cancer.

\begin{tabular}{|c|c|c|c|c|c|c|c|c|c|}
\hline Gene symbol & $\begin{array}{l}\text { Survival } \\
\text { outcome }\end{array}$ & $\begin{array}{c}\text { Basal } \\
\text { HR }(95 \% \mathrm{Cl})\end{array}$ & $p$-value & $\begin{array}{c}\text { Luminal A } \\
\text { HR (95\% Cl) }\end{array}$ & $p$-value & $\begin{array}{c}\text { Luminal B } \\
\text { HR (95\% Cl) }\end{array}$ & $p$-value & $\begin{array}{c}\text { HER2+ } \\
\text { HR }(95 \% \mathrm{CI})\end{array}$ & $p$-value \\
\hline \multirow[t]{4}{*}{ CAV1 } & os & 1.71 & 0.034 & 0.75 & 0.11 & 0.82 & 0.3 & 1.03 & 0.94 \\
\hline & RFS & 1.46 & 0.0034 & 0.75 & 0.0012 & 1.05 & 0.63 & 1.38 & 0.1 \\
\hline & DMFS & 1.34 & 0.24 & 0.93 & 0.64 & 1.12 & 0.51 & 1.62 & 0.13 \\
\hline & PPS & 1.62 & 0.11 & 1.03 & 0.89 & 1.06 & 0.78 & 0.66 & 0.28 \\
\hline \multirow[t]{4}{*}{ CAV2 } & OS & 1.58 & 0.071 & 0.76 & 0.14 & 0.76 & 0.15 & 0.56 & 0.081 \\
\hline & RFS & 1.39 & 0.011 & 0.79 & 0.0061 & 0.88 & 0.18 & 0.96 & 0.84 \\
\hline & DMFS & 1.46 & 0.13 & 0.94 & 0.67 & 0.99 & 0.95 & 0.97 & 0.93 \\
\hline & PPS & 1.81 & 0.049 & 1.08 & 0.68 & 1.21 & 0.39 & 0.65 & 0.25 \\
\hline \multirow[t]{4}{*}{ CAV3 } & OS & 0.83 & 0.44 & 0.65 & 0.019 & 0.92 & 0.68 & 0.6 & 0.13 \\
\hline & RFS & 0.81 & 0.11 & 0.77 & 0.0025 & 0.77 & 0.0075 & 0.66 & 0.036 \\
\hline & DMFS & 0.78 & 0.32 & 0.93 & 0.62 & 1.11 & 0.55 & 0.61 & 0.12 \\
\hline & PPS & 0.6 & 0.096 & 0.85 & 0.42 & 1.04 & 0.85 & 0.99 & 0.98 \\
\hline \multirow[t]{4}{*}{ CAVIN1 } & OS & 0.72 & 0.18 & 0.6 & 0.0052 & 1.11 & 0.59 & 0.88 & 0.69 \\
\hline & RFS & 0.93 & 0.55 & 0.71 & 0.00009 & 0.91 & 0.32 & 0.97 & 0.88 \\
\hline & DMFS & 0.9 & 0.66 & 0.92 & 0.55 & 0.99 & 0.98 & 1.99 & 0.035 \\
\hline & PPS & 0.95 & 0.86 & 0.8 & 0.27 & 1.19 & 0.43 & 0.95 & 0.9 \\
\hline \multirow[t]{4}{*}{ CAVIN2 } & OS & 1.33 & 0.38 & 0.53 & 0.015 & 0.63 & 0.18 & 0.6 & 0.21 \\
\hline & RFS & 0.99 & 0.95 & 0.59 & 0.000033 & 0.8 & 0.15 & 0.78 & 0.28 \\
\hline & DMFS & 0.87 & 0.69 & 0.84 & 0.54 & 0.95 & 0.88 & 0.79 & 0.53 \\
\hline & PPS & 1.13 & 0.78 & 1.21 & 0.51 & 1 & 0.99 & 1.53 & 0.34 \\
\hline \multirow[t]{4}{*}{ CAVIN3 } & OS & 0.94 & 0.8 & 0.88 & 0.48 & 1.29 & 0.18 & 1.22 & 0.54 \\
\hline & RFS & 1.41 & 0.0082 & 0.9 & 0.24 & 1.13 & 0.21 & 1.32 & 0.16 \\
\hline & DMFS & 1.11 & 0.68 & 1.08 & 0.61 & 1.226 & 0.19 & 1.59 & 0.15 \\
\hline & PPS & 0.75 & 0.34 & 0.87 & 0.49 & 1.38 & 0.15 & 0.92 & 0.82 \\
\hline \multirow[t]{4}{*}{ CAVIN4 } & OS & 0.71 & 0.29 & 1.04 & 0.89 & 1.19 & 0.62 & 1.87 & 0.13 \\
\hline & RFS & 0.88 & 0.43 & 1.17 & 0.21 & 0.92 & 0.61 & 1.66 & 0.028 \\
\hline & DMFS & 1.01 & 0.98 & 0.95 & 0.85 & 1.94 & 0.051 & 2 & 0.073 \\
\hline & PPS & 0.51 & 0.13 & 0.59 & 0.075 & 1.55 & 0.26 & 1.23 & 0.63 \\
\hline
\end{tabular}

Bold values mean $p$ value less than 0.05 . 
TABLE 4 | Correlation of CAVs/CAVINs transcription levels and survivals of patients with different HR/HER2 status of breast cancer.

\begin{tabular}{|c|c|c|c|c|c|c|c|c|c|c|c|}
\hline Gene symbol & Survival outcome & $\begin{array}{c}\text { HER2- } \\
\text { HR }(95 \% \mathrm{Cl})\end{array}$ & $p$-value & $\begin{array}{c}\text { PR+ } \\
\text { HR }(95 \% \mathrm{Cl})\end{array}$ & $p$-value & $\begin{array}{c}\text { PR- } \\
\text { HR (95\% Cl) }\end{array}$ & $p$-value & $\begin{array}{c}\text { ER+ } \\
\text { HR }(95 \% \mathrm{Cl})\end{array}$ & $p$-value & $\begin{array}{c}\text { ER- } \\
\text { HR }(95 \% \mathrm{Cl})\end{array}$ & $p$-value \\
\hline \multirow[t]{4}{*}{ CAV1 } & OS & 0.85 & 0.7 & 0.96 & 0.96 & 1.23 & 0.67 & 0.66 & 0.021 & 2.1 & 0.0017 \\
\hline & RFS & 0.97 & 0.84 & 0.83 & 0.3 & 1.19 & 0.23 & 0.81 & 0.012 & 1.62 & $3.20 \mathrm{E}-05$ \\
\hline & DMFS & 1.33 & 0.52 & 1.1 & 0.82 & 1.67 & 0.085 & 0.69 & 0.032 & 1.89 & 0.0042 \\
\hline & PPS & 0.39 & 0.07 & NA & NA & NA & NA & 0.7 & 0.08 & 1.37 & 0.24 \\
\hline \multirow[t]{4}{*}{ CAV2 } & OS & 0.97 & 0.95 & 0.89 & 0.86 & 0.86 & 0.76 & 0.64 & 0.013 & 1.39 & 0.15 \\
\hline & RFS & 0.93 & 0.57 & 0.8 & 0.22 & 1.26 & 0.13 & 0.77 & 0.0019 & 1.22 & 0.079 \\
\hline & DMFS & 1.32 & 0.53 & 1.31 & 0.52 & 1.67 & 0.084 & 0.69 & 0.03 & 1.56 & 0.043 \\
\hline & PPS & 1.09 & 0.87 & NA & NA & NA & NA & 0.77 & 0.21 & 1.4 & 0.2 \\
\hline \multirow[t]{4}{*}{ CAV3 } & OS & 0.68 & 0.42 & 5.12 & 0.032 & 1.13 & 0.8 & 0.85 & 0.37 & 0.62 & 0.049 \\
\hline & RFS & 1.16 & 0.26 & 0.9 & 0.55 & 1.13 & 0.41 & 0.93 & 0.41 & 1.01 & 0.92 \\
\hline & DMFS & 0.93 & 0.86 & 1.39 & 0.44 & 1.11 & 0.72 & 1.29 & 0.13 & 0.82 & 0.35 \\
\hline & PPS & 0.76 & 0.6 & NA & NA & NA & NA & 1.01 & 0.96 & 0.76 & 0.3 \\
\hline \multirow[t]{4}{*}{ CAVIN1 } & OS & 0.89 & 0.79 & 0.34 & 0.13 & 1.71 & 0.26 & 0.68 & 0.034 & 0.9 & 0.65 \\
\hline & RFS & 0.93 & 0.57 & 0.98 & 0.93 & 1.12 & 0.46 & 0.85 & 0.046 & 1.07 & 0.55 \\
\hline & DMFS & 1.06 & 0.9 & 1.17 & 0.7 & 1.66 & 0.091 & 0.84 & 0.29 & 1.32 & 0.21 \\
\hline & PPS & 0.54 & 0.23 & NA & NA & NA & NA & 0.8 & 0.29 & 0.85 & 0.53 \\
\hline \multirow[t]{4}{*}{ CAVIN2 } & OS & 1.55 & 0.42 & NA & NA & NA & NA & 1.11 & 0.79 & 1.26 & 0.52 \\
\hline & RFS & 0.52 & 2.80E-05 & 0.58 & 0.006 & 0.86 & 0.42 & 0.68 & 0.01 & 1.05 & 0.77 \\
\hline & DMFS & 0.4 & 0.17 & 0.34 & 0.093 & 1.04 & 0.92 & 0.59 & 0.27 & 1.07 & 0.87 \\
\hline & PPS & 2.48 & 0.095 & NA & NA & NA & NA & 1.68 & 0.25 & 1.73 & 0.18 \\
\hline \multirow[t]{4}{*}{ CAVIN3 } & OS & 1.09 & 0.84 & 0.61 & 0.46 & 1.35 & 0.52 & 1.05 & 0.79 & 1.25 & 0.33 \\
\hline & RFS & 1.29 & 0.056 & 1.07 & 0.71 & 1.5 & 0.007 & 0.98 & 0.79 & 1.47 & 8.60E-04 \\
\hline & DMFS & 0.91 & 0.84 & 1.15 & 0.74 & 1.41 & 0.25 & 0.95 & 0.78 & 1.32 & 0.2 \\
\hline & PPS & 0.56 & 0.26 & NA & NA & NA & NA & 1.24 & 0.3 & 1.01 & 0.97 \\
\hline \multirow[t]{4}{*}{ CAVIN4 } & OS & 0.66 & 0.44 & $N A$ & NA & NA & NA & 0.75 & 0.46 & 0.58 & 0.13 \\
\hline & RFS & 1.25 & 0.14 & 1.35 & 0.12 & 1.14 & 0.48 & 1.26 & 0.12 & 1.04 & 0.82 \\
\hline & DMFS & 1.11 & 0.87 & 0.88 & 0.83 & 1.66 & 0.18 & 0.84 & 0.71 & 1.35 & 0.48 \\
\hline & PPS & 0.84 & 0.76 & NA & NA & NA & NA & 1.54 & 0.35 & 1.03 & 0.94 \\
\hline
\end{tabular}

Bold values mean $p$ value less than 0.05 .

the gene ontology (GO) and Kyoto Encyclopedia of Genes and Genomes (KEGG) in the Database for Annotation, Visualization and Integrated Discovery (DAVID) (https://david.ncifcrf.gov/ summary.jsp). In GO enrichment analysis, we can obtain prediction of the functional roles of these genes on biological processes, cellular components, and molecular functions. Our results showed that GO:0007155 (cell adhesion), GO:0007160 (cell-matrix adhesion), GO:0006360 (transcription from RNA polymerase I promoter), and GO:0007179 (transforming growth factor beta receptor signaling pathway) were significantly regulated by CAVs and CAVINs alterations in breast adenocarcinoma (Figure 5A). GO:0005578 (proteinaceous extracellular matrix) and GO:0005201 (extracellular matrix structural constituent) were significantly influenced by alterations of caveolae-related genes (Figures 5B, C). These results indicated that the major function of CAVs and CAVINs mainly associate with cell surface components and their related molecular pathways.

Next, we conducted KEGG analysis to explore the pathways related to CAVs and CAVINs altered functions and the frequently altered neighbor genes. The results showed 20 pathways related to the functions of CAVs and CAVINs alterations in breast adenocarcinoma through (Figure 6A). Among these pathways, cfa04512: ECM-receptor interaction, cfa04510: focal adhesion, cfa04151: PI3K-Akt signaling pathway, and cfa05200: Pathways in cancer were prominently
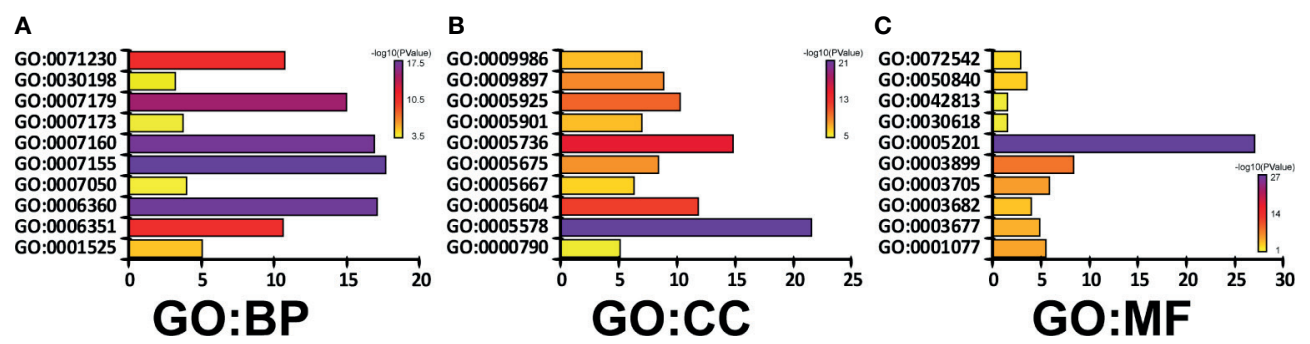

FIGURE 5 | Genes significantly associated with CAVs and CAVINs alterations indicated by GO enrichment as (A) biological processes, (B) cellular components, and (C) molecular functions. 
involved in the tumorigenesis and progression of breast cancer (Figures 6A, B).

Based on the survival results, we then implemented Gene Set Enrichment Analysis (GSEA) analysis to explore the relevant signaling pathways of CAVIN2 high expression group and low expression group of patients with breast cancer patients. Our results revealed significant relations between the expressions of several cancer related signaling pathways such as Wnt/ $\beta$-catenin signaling pathway, MAPK signaling pathway, E2F targets, G2M checkpoint components, and others related genes in breast carcinoma (Figure 7). The highly enriched gene sets in high expression group included $\mathrm{Wnt} / \beta$-catenin signaling pathway $(\mathrm{NES}=1.75, p=0.005)$, MAPK signaling pathway $(\mathrm{NES}=1.46$, $p=0.018)$, response to estradiol $(\mathrm{NES}=1.80, p=0)$, and regulation of estradiol on apoptosis of epithelial cells $(\mathrm{NES}=1.73, p=0.002)$. In low expression group, the highly enriched gene sets included E2F targets (NES $=-2.32, p=0$ ), G2M checkpoints (NES=-2.01, $p=0$ ), transcription factors sets in basal-like (NES=-1.99, $p=0)$, and P53 signaling pathway (NES=-1.67, $p=0.006$ ) (Figure 7).

\section{DISCUSSION}

Dysregulations of CAVs and CAVINs have been studied in many diseases, but few of them focused on the tumorigenesis,

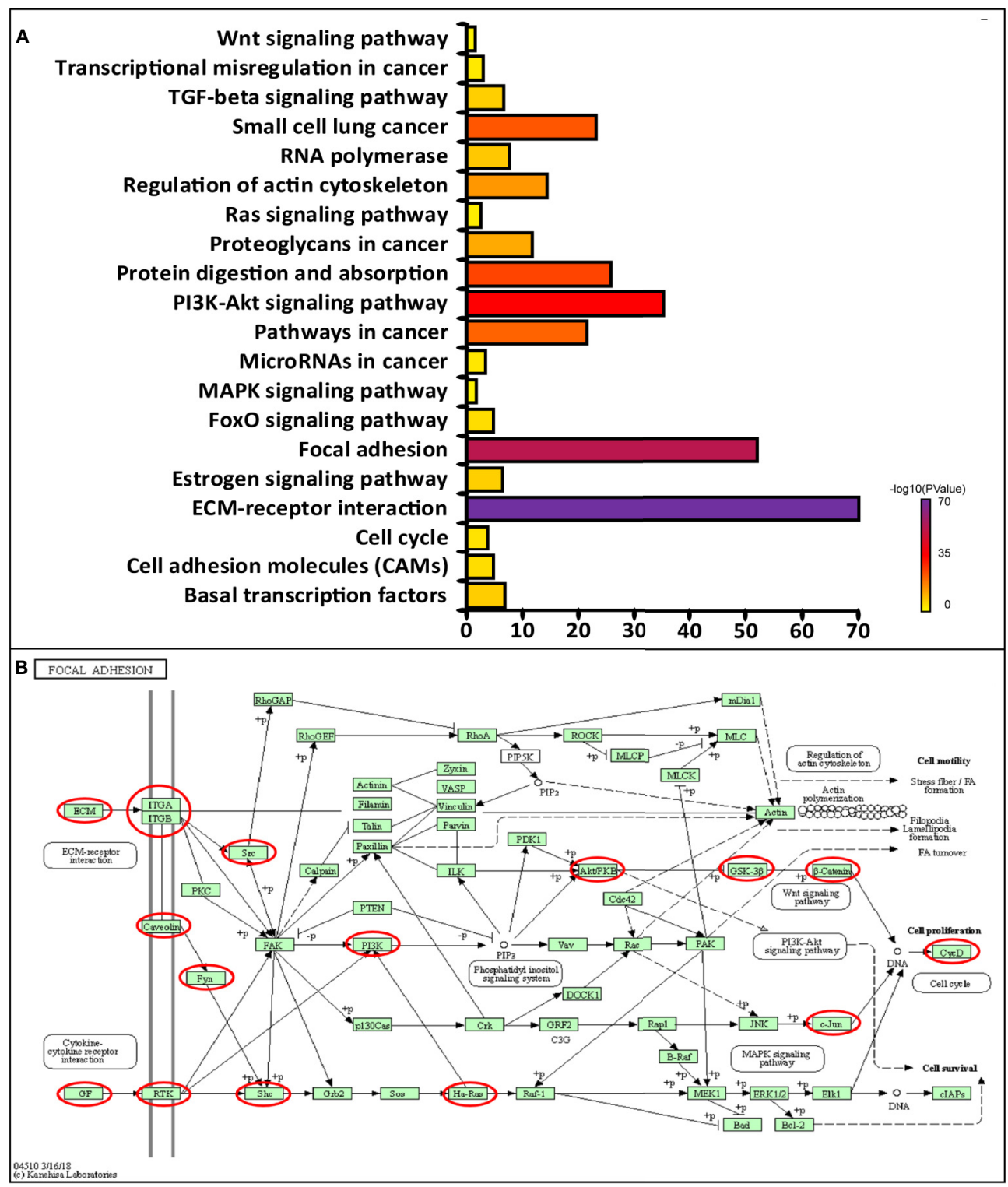

FIGURE 6 | (A) Molecular pathways associated with CAVs and CAVINs regulation implemented by DAVID tools. (B) Focal adhesion pathways regulated by CAVs and CAVINs in breast cancer. 

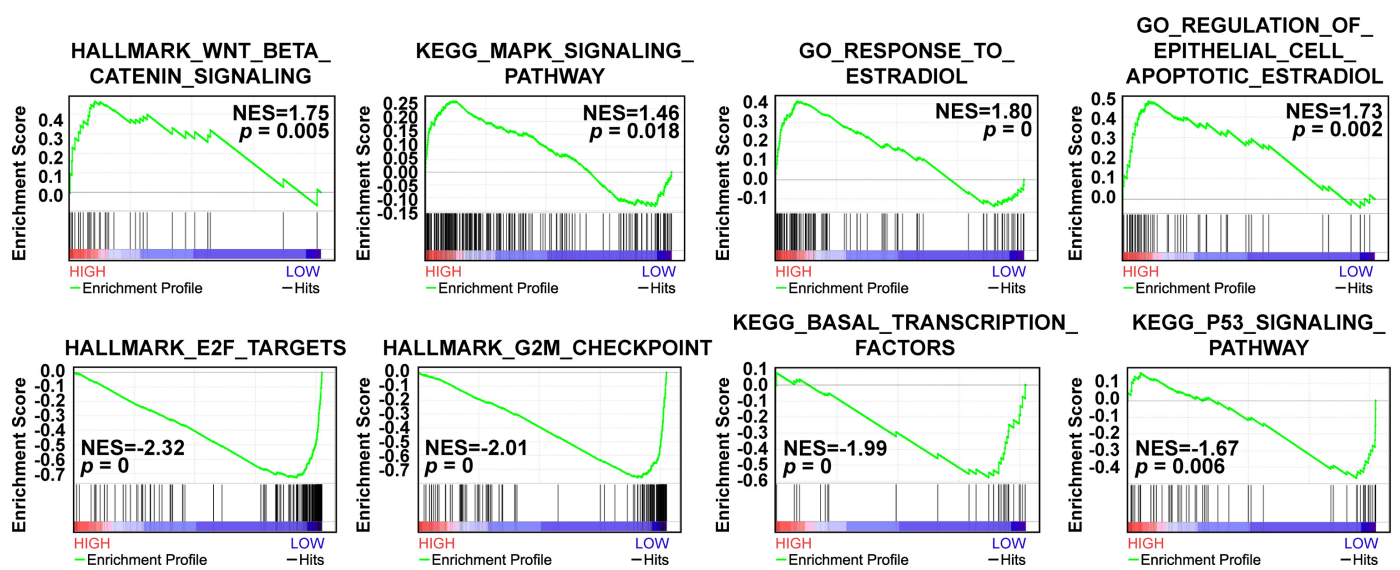

FIGURE 7 | GSEA analysis of altered CAVIN2 expression levels and relevant molecular pathways.

progression, and prognosis of cancer. Our study is the first to comprehensively investigate the expressions and prognosis of all caveolae-related proteins in patients with breast cancer. These findings are expected to provide solid evidences for target therapy towards breast cancer in the future, from which patients can obtain better outcomes and longer survival durations.

According to the genomic sequencing results from several investigated database, caveolae-related proteins all exhibited downregulation in breast cancer tissues except for CAVIN3. This indicated that most of CAVs and CAVINs acted as tumor suppressors in breast cancer development. However, we can conclude from Oncomine results that only CAVIN2 were downregulated in most types of cancers, while the expression levels of others were significantly elevated in specific types of cancers such as kidney cancer and lymphoma. From this prospective, CAV1, CAV2, and CAVIN1 can also play important roles in oncogenesis. The dual role of these proteins will need more investigations focusing organ-specific tumorigenesis in the future.

In terms of genes that code caveolins, CAV1 have been studied the most. Our investigation revealed that it was statistically less expressed in breast cancer tissues, which was elucidated by several databases as well as our immunohistochemistry results. It can be also correlated with OS and RFS of patients with breast cancer. The alteration rate in breast cancer was the highest among all the caveolae-related genes. Considering CAV1's association with breast cancer stem cell enrichment, these results implied it as a potential predictor of breast cancer progression. But as the most essential component of caveolae structure, the molecular mechanisms underlying the relationship between its downregulation and poor prognosis of patients with breast cancer need to be further understood.

Comparatively, CAV2 and CAV3 were less studied in cancer, but CAV3 have showed a better potential in correlating to breast cancer development. As a sub-structure of caveolae, CAV3 only revealed association with CAVIN4, which were both little expressed in cancer cells. Although intrafamily cross-analysis of survival curves indicated that CAV3 could be an independent predictor in regardless of the expression levels of other CAVs (Figure S4), evidences should be further consolidated to indicate whether it could accurately evaluate the risk of patients with breast cancer after receiving treatments.

The comprehensive analysis of cavins' expressions and functions had provided evidences of significant roles of CAVIN1 and CAVIN2 in inhibiting breast cancer development. Both CAVIN1 and CAVIN2 were significantly downregulated in breast cancer tissues and were associated with prognosis of patients. Previous studies have reported that loss of CAVIN1 induced a reduction in numbers of caveolae (32), and CAVIN1 knockout mice demonstrated a lack of caveolae, glucose intolerance and disorders in lungs and cardiovascular system (51-53). As a consequence, therapeutic methods targeting CAVIN1 may induce complications in cancer patients. Compared to CAVIN1, CAVIN2 demonstrated a better potential to serve as a therapeutic target. Downregulation of CAVIN2 in breast cancer tissues was the most significant of all caveolae-related genes, and it could assess prognostic values of patients with breast cancer in regardless of all others CAVINs (Figure S4). As previous studies have proved that downregulation of CAVIN2 could cause reduction in CAVIN1 and CAV1 expression (54), the interdependency of these 3 molecules makes CAVIN2 as a prominent therapeutic target in breast cancer treatment.

Furthermore, an important question raised from our current study is that which is more accurate in terms of results concluded from public database and real-world experiments. As in Figure 1, the results concluded from Oncomine database were not based on paired samples from patients with breast cancer. While in Figure 3, we have presented the most representative images for IHC experiments, which were derived from 20 paired samples from patients with breast cancer. Based on our current knowledge, Oncomine analysis and TCGA data just represent an average level of transcription or protein expression, which should be less accurate than real-world validation using paired samples. However, the amount of samples we used for IHC was 
not enough to compare with the very large scale study as public data. As a consequence, we believe that the Oncomine analysis and TCGA data should be more accurate in our current work. In our future work, multiple cohorts of samples from different hospitals at a larger amount will be considered to validate public data as well as the results presented by our experiments.

Meanwhile, predictions on neighbouring pathways of caveolae-related genes implied that the locations of CAVs and CAVINs were closely relevant to their functions, as the altered levels of these genes mainly influence molecules and pathways involved in cell adhesion and extracellular matrix function. Future studies can focus on the interactions between CAVs, CAVINs, proteins of extracellular matrix, and cell surface receptors, to further uncover the association of caveolae-related genes to the biological properties of cancer cells.

In conclusion, in this study we comprehensively analyzed the expression levels and prognostic values of caveolae-related genes in breast cancer, and provided an overview of their related molecular pathways and biological properties by investigating genomic sequencing data in several online databases. These results elucidated that most of CAVs and CAVINs act as suppressors in breast cancer tumorigenesis, and CAVIN2 were closely related to evaluating the risk of patients with breast cancer. Our findings suggested CAVIN2 could be potential targets for breast cancer therapy. Its downregulation could be a promising predictor for assessing prognostic values of breast cancer.

\section{DATA AVAILABILITY STATEMENT}

The original contributions presented in the study are included in the article/Supplementary Material. Further inquiries can be directed to the corresponding authors.

\section{ETHICS STATEMENT}

The studies involving human participants were reviewed and approved by Institutional Review Board of Tianjin Medical University Cancer Institute and Hospital. Written informed consent for participation was

\section{REFERENCES}

1. Anderson RG. The Caveolae Membrane System. Annu Rev Biochem (1998) 67:199-225. doi: 10.1146/annurev.biochem.67.1.199

2. Stan RV. Structure of Caveolae. Biochim Biophys Acta (2005) 1746:334-48. doi: 10.1016/j.bbamcr.2005.08.008

3. Parton RG, Simons K. The Multiple Faces of Caveolae. Nat Rev Mol Cell Biol (2007) 8:185-94. doi: 10.1038/nrm2122

4. Harris J, Werling D, Hope JC, Taylor G, Howard CJ. Caveolae and Caveolin in Immune Cells: Distribution and Functions. Trends Immunol (2002) 23:15864. doi: 10.1016/S1471-4906(01)02161-5

5. Vykoukal J, Fahrmann JF, Gregg JR, Tang Z, Basourakos S, Irajizad E, et al. Caveolin-1-Mediated Sphingolipid Oncometabolism Underlies a Metabolic Vulnerability of Prostate Cancer. Nat Commun (2020) 11:4279. doi: 10.1038/ s41467-020-17645-z not required for this study in accordance with the national legislation and the institutional requirements.

\section{AUTHOR CONTRIBUTIONS}

Conceptualization, YT. Investigation, YT, XL, and YY. Writing Original Draft, YT and XL. Writing - Review \& Editing, YT, XL, JH, HZ, BW, YL, LF, RS, and YY. Visualization, YT, XL, RS, and YY. Supervision, RS and YY. Funding Acquisition, XL and YY. All authors contributed to the article and approved the submitted version.

\section{FUNDING}

This study was funded by the National Natural Science Foundation of China (No. 82172827 and 81502518) and Tianjin Bureau of Foreign Experts Affairs JH-2018007 0802-2018038.

\section{SUPPLEMENTARY MATERIAL}

The Supplementary Material for this article can be found online at: https://www.frontiersin.org/articles/10.3389/fonc.2021. 703501/full\#supplementary-material

Supplementary Figure 1 | Overall survival (OS), relapse-free survival (RFS), distant metastasis-free survival (DMFS), and post progression survival (PPS) curves of patients with breast cancer under different expression levels of CAVs and CAVINs.

Supplementary Figure 2 | OS, RFS, DMFS, and PPS curves of patients with different molecular subtypes of breast cancer divided by different expression levels of CAVs and CAVINs.

Supplementary Figure 3 | OS, RFS, DMFS, and PPS curves of patients with different receptor status of breast cancer divided by different expression levels of CAVs and CAVINs.

Supplementary Figure 4 I Intra-family cross analysis of different CAVs and CAVINs levels on RFS of patients with breast cancer.

6. Meng F, Joshi B, Nabi IR. Galectin-3 Overrides PTRF/Cavin-1 Reduction of PC3 Prostate Cancer Cell Migration. PLoS One (2015) 10:e0126056. doi: 10.1371/journal.pone.0126056

7. Romano A, Feola A, Porcellini A, Gigantino V, Di Bonito M, Di Mauro A, et al. Estrogen Induces Selective Transcription of Caveolin1 Variants in Human Breast Cancer Through Estrogen Responsive Element-Dependent Mechanisms. Int J Mol Sci (2020) 21:5989. doi: 10.3390/ijms21175989

8. Ravid D, Maor S, Werner H, Liscovitch M. Caveolin-1 Inhibits Cell Detachment-Induced P53 Activation and Anoikis by Upregulation of Insulin-Like Growth Factor-I Receptors and Signaling. Oncogene (2005) 24:1338-47. doi: 10.1038/sj.onc. 1208337

9. Shi Y, Tan SH, Ng S, Zhou J, Yang ND, Koo GB, et al. Critical Role of CAV1/ Caveolin-1 in Cell Stress Responses in Human Breast Cancer Cells via Modulation of Lysosomal Function and Autophagy. Autophagy (2015) 11:76984. doi: 10.1080/15548627.2015.1034411 
10. Tian Y, Yu Y, Hou LK, Chi JR, Mao JF, Xia L, et al. Serum Deprivation Response Inhibits Breast Cancer Progression by Blocking Transforming Growth Factor-Beta Signaling. Cancer Sci (2016) 107:274-80. doi: 10.1111/ cas. 12879

11. Philips BJ, Kumar A, Burki S, Ryan JP, Noda K, D’Cunha J. TriptolideInduced Apoptosis in Non-Small Cell Lung Cancer via a Novel Mir204-5p/ Caveolin-1/Akt-Mediated Pathway. Oncotarget (2020) 11:2793-806. doi: 10.18632/oncotarget.27672

12. Feng Y, Jiang W, Zhao W, Lu Z, Gu Y, Dong Y. Mir-124 Regulates Liver Cancer Stem Cells Expansion and Sorafenib Resistance. Exp Cell Res (2020) 394:112162. doi: 10.1016/j.yexcr.2020.112162

13. Liu F, Shangli Z, Hu Z. CAV2 Promotes the Growth of Renal Cell Carcinoma Through the EGFR/PI3K/Akt Pathway. Onco Targets Ther (2018) 11:6209-16. doi: $10.2147 /$ OTT.S172803

14. Kitowska A, Wesserling M, Seroczynska B, Szutowicz A, Ronowska A, Peksa $\mathrm{R}$, et al. Differentiation of High-Risk Stage I and II Colon Tumors Based on Evaluation of CAV1 Gene Expression. J Surg Oncol (2015) 112:408-14. doi: $10.1002 /$ jso.23995

15. Shao S, Qin T, Qian W, Yue Y, Xiao Y, Li X, et al. Positive Feedback in Cav-1ROS Signalling in Pscs Mediates Metabolic Coupling Between Pscs and Tumour Cells. J Cell Mol Med (2020). doi: 10.1111/jcmm.15596

16. Liang C, Shi S, Meng Q, Liang D, Hua J, Qin Y, et al. Mir-29a, Targeting Caveolin 2 Expression, Is Responsible for Limitation of Pancreatic Cancer Metastasis in Patients With Normal Level of Serum CA125. Int J Cancer (2018) 143:2919-31. doi: 10.1002/ijc.31654

17. Sung H, Ferlay J, Siegel RL, Laversanne M, Soerjomataram I, Jemal A, et al. Global Cancer Statistics 2020: GLOBOCAN Estimates of Incidence and Mortality Worldwide for 36 Cancers in 185 Countries. CA Cancer J Clin (2021) 71:209-49. doi: 10.3322/caac.21660

18. Sorlie T, Perou CM, Tibshirani R, Aas T, Geisler S, Johnsen H, et al. Gene Expression Patterns of Breast Carcinomas Distinguish Tumor Subclasses With Clinical Implications. Proc Natl Acad Sci U S A (2001) 98:10869-74. doi: 10.1073/pnas.191367098

19. Johnston SR. Clinical Efforts to Combine Endocrine Agents With Targeted Therapies Against Epidermal Growth Factor Receptor/Human Epidermal Growth Factor Receptor 2 and Mammalian Target of Rapamycin in Breast Cancer. Clin Cancer Res (2006) 12:1061s-8s. doi: 10.1158/1078-0432.CCR-052125

20. Cao L, Niu Y. Triple Negative Breast Cancer: Special Histological Types and Emerging Therapeutic Methods. Cancer Biol Med (2020) 17:293-306. doi: 10.20892/j.issn.2095-3941.2019.0465

21. Williams TM, Lisanti MP. The Caveolin Proteins. Genome Biol (2004) 5:214. doi: 10.1186/gb-2004-5-3-214

22. Shatz M, Liscovitch M. Caveolin-1: A Tumor-Promoting Role in Human Cancer. Int J Radiat Biol (2008) 84:177-89. doi: 10.1080/09553000701745293

23. Williams TM, Lisanti MP. The Caveolin Genes: From Cell Biology to Medicine. Ann Med (2004) 36:584-95. doi: 10.1080/07853890410018899

24. Parton RG. Caveolae Meet Endosomes: A Stable Relationship? Dev Cell (2004) 7:458-60. doi: 10.1016/j.devcel.2004.09.009

25. Wang S, Wang N, Zheng Y, Yang B, Liu P, Zhang F, et al. Caveolin-1 Inhibits Breast Cancer Stem Cells via C-Myc-Mediated Metabolic Reprogramming. Cell Death Dis (2020) 11:450. doi: 10.1038/s41419-020-2667-x

26. Kang J, Park JH, Lee HJ, Jo U, Park JK, Seo JH, et al. Caveolin-1 Modulates Docetaxel-Induced Cell Death in Breast Cancer Cell Subtypes Through Different Mechanisms. Cancer Res Treat (2016) 48:715-26. doi: 10.4143/ crt.2015.227

27. Scherer PE, Lewis RY, Volonte D, Engelman JA, Galbiati F, Couet J, et al. CellType and Tissue-Specific Expression of Caveolin-2. Caveolins 1 and 2 Co-Localize and Form a Stable Hetero-Oligomeric Complex. vivo J Biol Chem (1997) 272:29337-46. doi: 10.1074/jbc.272.46.29337

28. Shatseva T, Lee DY, Deng Z, Yang BB. Microrna Mir-199a-3p Regulates Cell Proliferation and Survival by Targeting Caveolin-2. J Cell Sci (2011) 124:282636. doi: $10.1242 /$ jcs. 077529

29. Tang Z, Scherer PE, Okamoto T, Song K, Chu C, Kohtz DS, et al. Molecular Cloning of Caveolin-3, A Novel Member of the Caveolin Gene Family Expressed Predominantly in Muscle. J Biol Chem (1996) 271:2255-61. doi: $10.1074 /$ jbc.271.4.2255
30. Wanaski SP, Ng BK, Glaser M. Caveolin Scaffolding Region and the Membrane Binding Region of SRC Form Lateral Membrane Domains. Biochemistry (2003) 42:42-56. doi: 10.1021/bi012097n

31. Yi JS, Mun DG, Lee H, Park JS, Lee JW, Lee JS, et al. PTRF/Cavin-1 Is Essential for Multidrug Resistance in Cancer Cells. J Proteome Res (2013) 12:605-14. doi: $10.1021 / \mathrm{pr} 300651 \mathrm{~m}$

32. Gould ML, Williams G, Nicholson HD. Changes in Caveolae, Caveolin, and Polymerase 1 and Transcript Release Factor (PTRF) Expression in Prostate Cancer Progression. Prostate (2010) 70:1609-21. doi: 10.1002/ pros. 21195

33. Hansen CG, Bright NA, Howard G, Nichols BJ. SDPR Induces Membrane Curvature and Functions in the Formation of Caveolae. Nat Cell Biol (2009) 11:807-14. doi: $10.1038 /$ ncb 1887

34. Ozturk S, Papageorgis P, Wong CK, Lambert AW, Abdolmaleky HM, Thiagalingam A, et al. SDPR Functions as a Metastasis Suppressor in Breast Cancer by Promoting Apoptosis. Proc Natl Acad Sci U S A (2016) 113:638-43. doi: 10.1073/pnas.1514663113

35. McMahon KA, Zajicek H, Li WP, Peyton MJ, Minna JD, Hernandez VJ, et al. SRBC/Cavin-3 Is a Caveolin Adapter Protein That Regulates Caveolae Function. EMBO J (2009) 28:1001-15. doi: 10.1038/ emboj.2009.46

36. Xu XL, Wu LC, Du F, Davis A, Peyton M, Tomizawa Y, et al. Inactivation of Human SRBC, Located Within the 11p15.5-P15.4 Tumor Suppressor Region, in Breast and Lung Cancers. Cancer Res (2001) 61:7943-9.

37. Tong SY, Ki KD, Lee JM, Kang MJ, Ha TK, Chung SI, et al. Frequent Inactivation of Hsrbc in Ovarian Cancers by Promoter Cpg Island Hypermethylation. Acta Obstet Gynecol Scand (2010) 89:629-35. doi: 10.3109/ 00016341003678443

38. Faggi F, Codenotti S, Poliani PL, Cominelli M, Chiarelli N, Colombi M, et al. MURC/Cavin-4 Is Co-Expressed With Caveolin-3 in Rhabdomyosarcoma Tumors and Its Silencing Prevents Myogenic Differentiation in the Human Embryonal RD Cell Line. PLoS One (2015) 10:e0130287. doi: 10.1371/ journal.pone. 0130287

39. Tang Z, Li C, Kang B, Gao G, Li C, Zhang Z. GEPIA: A Web Server for Cancer and Normal Gene Expression Profiling and Interactive Analyses. Nucleic Acids Res (2017) 45:W98-102. doi: 10.1093/nar/gkx247

40. Cancer Genome Atlas N. Comprehensive Molecular Portraits of Human Breast Tumours. Nature (2012) 490:61-70. doi: 10.1038/nature11412

41. Gyorffy B, Surowiak P, Budczies J, Lanczky A. Online Survival Analysis Software to Assess the Prognostic Value of Biomarkers Using Transcriptomic Data in Non-Small-Cell Lung Cancer. PLoS One (2013) 8:e82241. doi: 10.1371/ journal.pone.0082241

42. Sorlie T, Tibshirani R, Parker J, Hastie T, Marron JS, Nobel A, et al. Repeated Observation of Breast Tumor Subtypes in Independent Gene Expression Data Sets. Proc Natl Acad Sci U S A (2003) 100:8418-23. doi: 10.1073/ pnas. 0932692100

43. Curtis C, Shah SP, Chin SF, Turashvili G, Rueda OM, Dunning MJ, et al. The Genomic and Transcriptomic Architecture of 2,000 Breast Tumours Reveals Novel Subgroups. Nature (2012) 486:346-52. doi: 10.1038/ nature 10983

44. Richardson AL, Wang ZC, De Nicolo A, Lu X, Brown M, Miron A, et al. X Chromosomal Abnormalities in Basal-Like Human Breast Cancer. Cancer Cell (2006) 9:121-32. doi: 10.1016/j.ccr.2006.01.013

45. Perou CM, Sorlie T, Eisen MB, van de Rijn M, Jeffrey SS, Rees CA, et al. Molecular Portraits of Human Breast Tumours. Nature (2000) 406:747-52. doi: $10.1038 / 35021093$

46. Zhao H, Langerod A, Ji Y, Nowels KW, Nesland JM, Tibshirani R, et al. Different Gene Expression Patterns in Invasive Lobular and Ductal Carcinomas of the Breast. Mol Biol Cell (2004) 15:2523-36. doi: 10.1091/ mbc.e03-11-0786

47. Ma XJ, Dahiya S, Richardson E, Erlander M, Sgroi DC. Gene Expression Profiling of the Tumor Microenvironment During Breast Cancer Progression. Breast Cancer Res (2009) 11:R7. doi: 10.1186/bcr2222

48. Radvanyi L, Singh-Sandhu D, Gallichan S, Lovitt C, Pedyczak A, Mallo G, et al. The Gene Associated With Trichorhinophalangeal Syndrome in Humans Is Overexpressed in Breast Cancer. Proc Natl Acad Sci U S A (2005) 102:11005-10. doi: 10.1073/pnas.0500904102 
49. Finak G, Bertos N, Pepin F, Sadekova S, Souleimanova M, Zhao H, et al. Stromal Gene Expression Predicts Clinical Outcome in Breast Cancer. Nat Med (2008) 14:518-27. doi: 10.1038/nm1764

50. Gluck S, Ross JS, Royce M, McKenna EF Jr., Perou CM, Avisar E, et al. TP53 Genomics Predict Higher Clinical and Pathologic Tumor Response in Operable Early-Stage Breast Cancer Treated With Docetaxel-Capecitabine +/- Trastuzumab. Breast Cancer Res Treat (2012) 132:781-91. doi: 10.1007/s10549-011-1412-7

51. Liu L, Brown D, McKee M, Lebrasseur NK, Yang D, Albrecht KH, et al. Deletion of Cavin/PTRF Causes Global Loss of Caveolae, Dyslipidemia, and Glucose Intolerance. Cell Metab (2008) 8:310-7. doi: 10.1016/j.cmet. 2008.07.008

52. Govender P, Romero F, Shah D, Paez J, Ding SY, Liu L, et al. Cavin1; A Regulator of Lung Function and Macrophage Phenotype. PLoS One (2013) 8: e62045. doi: 10.1371/journal.pone.0062045

53. Low JY, Nicholson HD. Emerging Role of Polymerase-1 and Transcript Release Factor (PTRF/Cavin-1) in Health and Disease. Cell Tissue Res (2014) 357:505-13. doi: 10.1007/s00441-014-1964-z

54. Mineo C, Ying YS, Chapline C, Jaken S, Anderson RG. Targeting of Protein Kinase Calpha to Caveolae. J Cell Biol (1998) 141:601-10. doi: 10.1083/ jcb.141.3.601
Conflict of Interest: The authors declare that the research was conducted in the absence of any commercial or financial relationships that could be construed as a potential conflict of interest.

The reviewer XZ declared a shared affiliation, with no collaboration, with the authors to the handling editor at the time of the review.

Publisher's Note: All claims expressed in this article are solely those of the authors and do not necessarily represent those of their affiliated organizations, or those of the publisher, the editors and the reviewers. Any product that may be evaluated in this article, or claim that may be made by its manufacturer, is not guaranteed or endorsed by the publisher.

Copyright $\odot 2021$ Tian, Liu, Hu, Zhang, Wang, Li, Fu, Su and Yu. This is an openaccess article distributed under the terms of the Creative Commons Attribution License (CC BY). The use, distribution or reproduction in other forums is permitted, provided the original author(s) and the copyright owner(s) are credited and that the original publication in this journal is cited, in accordance with accepted academic practice. No use, distribution or reproduction is permitted which does not comply with these terms. 